\title{
Retos y soluciones de ingeniería en el diseño de un re-entry exploratorio desde el revestimiento intermedio en la cuenca sub-andina colombiana: caso de estudio
}

\section{Engineering challenges and solutions in the design of an exploratory re-entry from the intermediate casing in the Colombian sub-Andean basin: case study}

\author{
A. Montes-Humánez ${ }^{1 *}$; C. Sarmiento ${ }^{1} ;$ J. Tovar $^{1}$; W. Carreño ${ }^{1}$
}

Forma de citar: Montes-Humánez, A., Sarmiento, C., Tovar, J., \& Carreño, W. (2020). Retos y soluciones de ingeniería en el diseño de un re-entry exploratorio desde el revestimiento intermedio en la cuenca sub-andina colombiana: Caso de estudio. Revista Fuentes, el reventón energético, 19(1), 45-64. https://doi.org/10.18273/revfue.v19n1-2021005

\section{Resumen}

Las formaciones y estructuras en Niscota, campo en exploración en la cuenca sub-andina Colombiana, se caracterizan por su alta complejidad tectónica-geomecánica y sedimentológica, al igual que sus vecinos del sur, Pauto y Floreña. Esto conlleva a que los proyectos de exploración (y especialmente la perforación de pozos) sean costosos, demorados y con estructuras de riesgos con impactos elevados (evaluados en términos de tiempo y costo). Una solución para la reducción de costos en proyectos de exploración, es la re-utilización de pozos existentes mediante proyectos de reacceso con desplazamientos significativos, apuntando a direcciones distintas de la estructura en exploración. No obstante, este tipo de prospectos, conllevan a afrontar retos técnicos, operacionales y organizacionales, para los cuales se requiere un detalle de ingeniería y madurez organizacional importante.

En este artículo, presentamos un caso de estudio, en el cual se dio soluciones para los siguientes retos: Evaluación del punto de asentamiento de la cuchara de desviación y los riesgos asociados; evaluación del riesgo de fatiga de la sarta durante las operaciones de molienda y posteriores; manejo de recortes de metal de la ventana en revestimiento de 11.75 in.; mapa de parámetros de perforación de ventana en revestimiento de 11.75 in.; perforación en dirección ortogonal al buzamiento en zona fallada; perforación en 10.625 in. Con curvaturas mayores a $4.5^{\circ} / 100 \mathrm{ft}$ en roca dura e intercalada y manejo de incertidumbre geológica en zonas de baja resolución sísmica. Adicionalmente, se presentan los factores de éxito, desde el punto de vista de gerencia de proyecto y seguridad de procesos, que permitieron un valor ganado satisfactorio. Finalmente, las soluciones propuestas a los retos mencionados y la gestión eficiente del proyecto, permitieron lograr el menor tiempo no productivo (NPT) del campo incluso comparado con proyectos similares en los campos análogos del sur (hasta un 7\% menos), y ahorros de 1.7 millones de dólares.

Palabras clave: Reacceso, desviación de pozo original, curvatura, alto desplazamiento.

\begin{abstract}
The formations and structures in Niscota, a field under exploration in the Colombian sub-Andean basin, are characterized by their high tectonic-geomechanical and sedimentological complexity as their southern neighbors, Pauto and Floreña. This means that the exploration projects (especially the drilling of wells) are expensive, delayed, and with risk structures with high impacts (evaluated in terms of time and cost). A solution to reduce costs on exploration projects is to reuse existing wells through re-access projects with significant displacements, aiming to different directions of the structure under exploration. However, these types of prospects lead to face technical, operational, and organizational challenges, for which important engineering detail and organizational maturity are required.
\end{abstract}

In this article, we present a case study where it is provided the solutions for the following challenges: Evaluation of the settling point of the diversion bucket and the associated risks; assessing the risk of string fatigue during and after milling operations; 11.75 in. siding window metal trim handling; 11.75 in. cladding window drilling parameter map; drilling in a direction orthogonal to the dip in the fault zone, and drilling in 10.625 in with curvatures greater than 4.5\%100 ft in hard and interbedded rock along with the management of geological

\footnotetext{
${ }^{1}$ Equión-Energía.

"abrahamcmontes@javeriana.edu.co
} 
uncertainty in areas of low seismic resolution. Additionally, the success factors are presented from the project management perspective and process safety, which allowed satisfactory earned value. Finally, the solutions proposed to the previous challenges and the efficient management of the project allowed to achieve the lowest non-productive time (NPT) of the field even compared to similar projects on similar south fields (up to 7\% less) saving 1.7 million dollars.

Keywords: Re-entry, sidetrack, dogleg severity, curvature, high displacement, long step out.

\section{Introducción}

Contexto del campo. La cuenca sub-andina colombiana se ubica en el costado oriental de la cordillera, tal como se muestra en la figura 1. Este trabajo se centra en la zona oriental de la cuenca, conocida como el Piedemonte (Eastern foothills), tal como lo menciona la Agencia Nacional de Hidrocarburos (ANH, 2007) the basins of Colombia have undergone changes in direction and shape due to diff erent events of rifting and oblique collisions followed by transpression and transtensional tectonics deformation. As a consequence the tectonic evolution of most, if not all, Colombian basins should be considered as polyhistory. From a structural/stratigraphic perspective, our knowledge varies greatly from basin to basin. This complicates comparison, especially where an author has emphasized only one aspect of the basin evolution. The northwestern corner of South America, where Colombia is located, has experienced diff erent geologic events that controlled the distribution, genesis, basin fi 11 and bounding structures of the sedimentary basins. In this chapter, the chronology of the main geologic phenomena that occurred in the country are sketched, emphasizing those that generated sedimentary deposits that, nowadays, constitute source rocks, reservoir rocks, sealing units and overburden sequences and fi nally hydrocarbon traps. In order to describe these events it is necessary to point out that Colombia can be divided in at least three main tectonic domains: 1. A continuación, algunas propiedades de esta cuenca, que hacen la actividad exploratoria compleja:
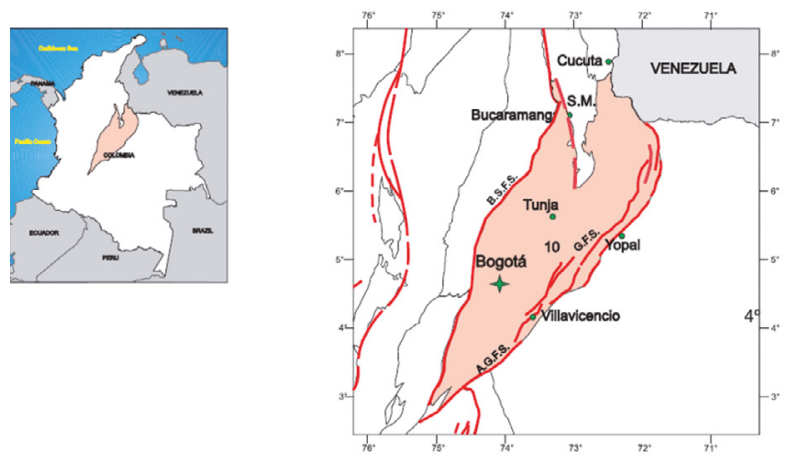

Figura 1. Ubicación geográfica de la cuenca sub-andina en la cordillera oriental (ANH, 2007).
1. Debido a la dinámica tectónica y la orogénesis, las formaciones que originalmente se depositaron de manera horizontal, han sido plegadas y por ende, en su mayoría falladas y fracturadas. En la figura 2 , puede observarse una sección que ejemplifica la trayectoria típica de un pozo en esta cuenca y la posición relativa de las formaciones y las estructuras en el subsuelo.

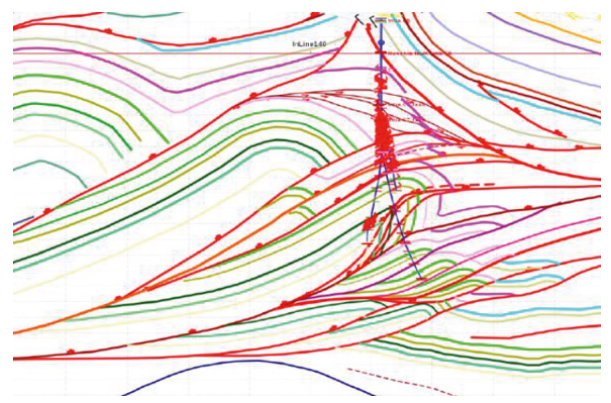

Figura 2. Sección transversal típica en la cuenca sub-andina, parte oriental.

2. Se aprecian ambientes de sedimentación con variaciones temporales importantes, por lo cual las formaciones tienen gradaciones sedimentológicas marcadas en intervalos cortos. En otras palabras, es posible encontrar en una misma formación, intercalaciones de sedimentos de varios tamaños. En la figura 3, se muestra la evaluación de la formación Los Cuervos, en la parte norte de la cuenca sub-andina colombiana. En la columna izquierda, se muestra con color verde el registro de rayos gamma. Por la interpretación litológica, esta formación contiene una combinación de intervalos de distinta naturaleza (mineralogía, resistencia compresiva, tamaño de sedimentos, entre otros). Cabe resaltar que, en términos generales, los intervalos arcillosos tienen más ductilidad que los intervalos arenosos y por tanto tienden a fallar por esfuerzo de cizalla y no por fractura. Adicionalmente, los intervalos arcillosos tienden a tener fisilidad, producto del metamorfismo, por el ambiente en el que han sido sometidos. Por ende, la producción de derrumbes producto de planos de debilidad en dichos intervalos es muy común. 


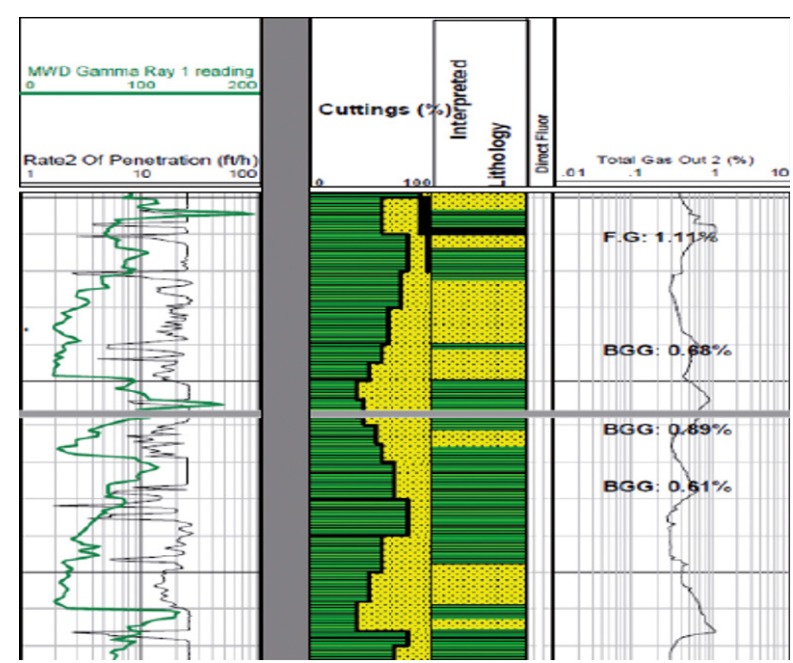

Figura 3. Registro de evaluación de formación (FEL) para la formación Los Cuervos.

Además de la naturaleza de la formación, está el ambiente tectónico altamente complejo, que implica incremento del riesgo de inestabilidad de pozo. De ahí a que una de las estrategias exploradas en el campo es tratar de perforar en dirección del esfuerzo horizontal máximo.

La consecuencia de esta naturaleza intercalada de sedimentos con cierto grado de metamorfismo (debido a la orogénesis), es la irregularidad del calibre del hueco perforado y la sensibilidad del calibre al tiempo de exposición del hoyo. La figura 4 muestra la geometría de un hueco perforado con broca de 8.5 pulgadas en una zona intercalada. Puede observarse la complejidad geométrica del hueco abierto (open hole) y la pobre calidad de cemento luego de haberlo revestido, como consecuencia de esto mismo.

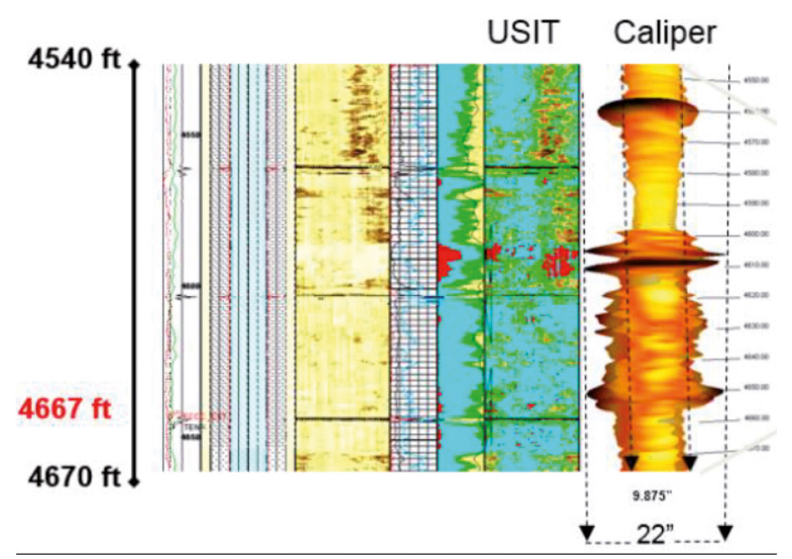

Figura 4. Degradación del calibre de una zona intercalada perforada en 8.5 ", formando cuellos de botella y escalones.

Para ilustrar la sensibilidad al tiempo de exposición, la figura 5 muestra el registro de rayos gamma del tope de una formación típicamente consolidada (resistencia compresiva uniaxial, o UCS $>20,000 \mathrm{psi}$ ), en cuyo tope se aprecian algunas arcillas intercaladas. En todos los casos se usó un peso de lodo entre 8.4 y 8.5 ppg. El pozo del lado derecho muestra un mayor grado de contenido arcilloso en el tope y aun así, menor degradación del calibre. La única diferencia entre ambos pozos, ha sido el tiempo de exposición del hueco.

Cabe aclarar que el ejemplo referido en la figura 5 no genera típicamente problemas de geometría relevantes (dada su alta resistencia compresiva), que justifiquen la operación de ensanchamiento del hueco. No es el caso de otras formaciones con intervalos arcillosos de menor resistencia, como la mencionada formación Los Cuervos, o los componentes de la unidad "Carbonera".

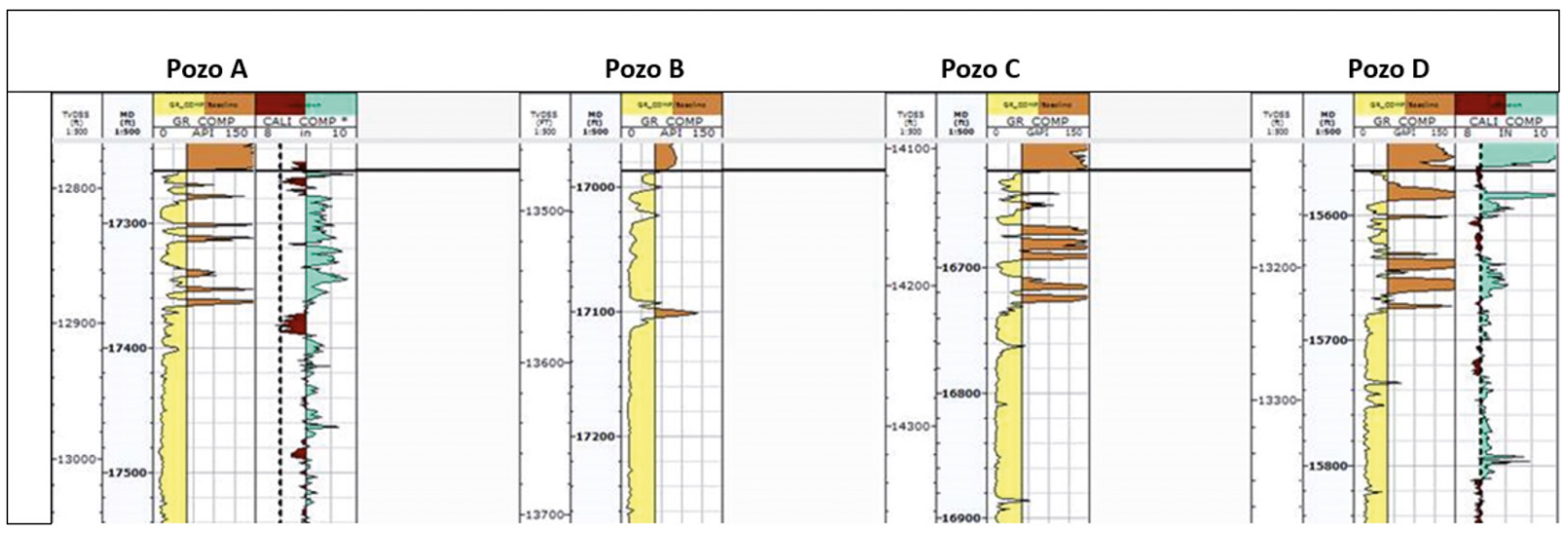

Figura 5. Degradación del calibre en zona intercalada de yacimiento debido al tiempo de exposición. El pozo A muestra mayor contenido de arena en la zona intercalada (alrededor de 30\%) y aún así, una mayor degradación del calibre, debido al tiempo de exposición. 
3. Formaciones yacimiento de altos espesores estratigráficos con altos impactos de abrasión y compresibilidades de hasta 30,000 psi. La perforación de estos intervalos es un reto en términos del diseño de brocas. Numerosos diseños han sido creados exclusivamente para la cuenca sub-andina en Suramérica. La perforación de estos intervalos además implica una dinámica de sarta compleja debido al alto torque reactivo y la resistencia compresiva. Por este motivo, es típico perforar estos intervalos a $2-3 \mathrm{ft} / \mathrm{h}$ promedio. La figura 6 muestra el desgaste de una broca que ha perforado únicamente $54 \mathrm{ft}$ de una formación yacimiento. A pesar de que el mecanismo de corte de una broca PDC se ajusta a una formación que falle por cizalla, este tipo de brocas han logrado el mejor desempeño sostenido hasta el momento con la tecnología de cortadores rotatorios de Schlumberger (Corridas >180 ft, ROP: $2-3 \mathrm{ft} / \mathrm{h}$ ). El éxito de esta tecnología, ha sido reportado por otros autores a nivel mundial (Teng et al., 2015; Zhang et al., 2013)which is far to meet the economic requirements of oil company, due to its unique drilling challenges caused by the highsteep structure (maximum dip angle $80^{\circ}$.
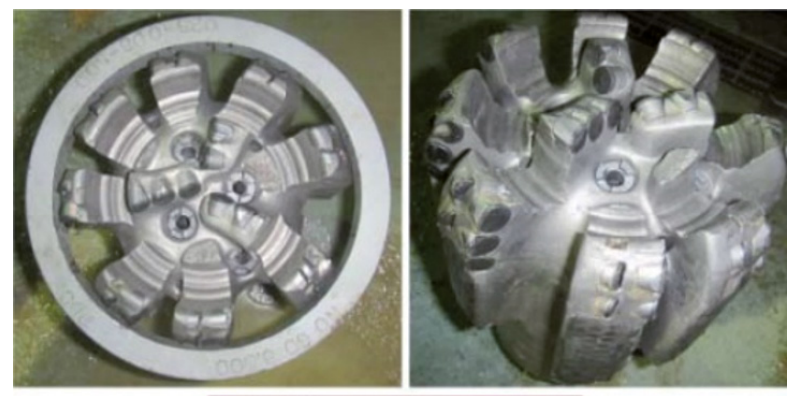

$81 / 2 * P D C$ Bit came out In-Gauge

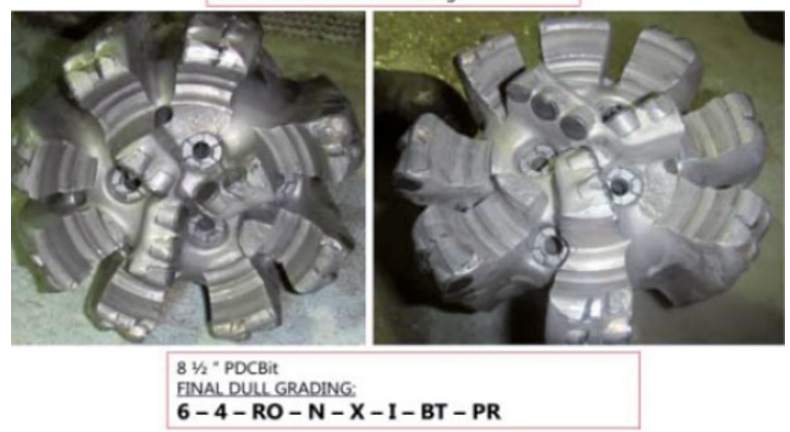

Figura 6. Desgaste de broca PDC que perforó $54 \mathrm{ft}$ de una formación altamente abrasiva y resistente.

4. Debido al componente estructural y tectónico de la cuenca, el modelamiento geomecánico de la estabilidad del agujero es complejo. Como resultado, se deben usar altos pesos de lodo para zonas potencialmente inestables, en la misma sección de hueco donde coexisten formaciones permeables ó depletadas; usando fluidos con distribuciones de tamaño de partícula diseñados para el puenteo de gargantas porales y planos de debilidad, además del alto contenido requerido de asfaltos y gilsonita.

5. Zonas con baja resolución sísmica.

6. La complejidad de las negociaciones sociales y las condiciones topográficas han dificultado la construcción de nuevas localizaciones por su alto costo (Del orden de 20 - 30 millones de dólares); por lo cual la campaña de exploración en esta cuenca ha ido evolucionando progresivamente a pozos de mayor desplazamiento hasta del orden de $6000 \mathrm{ft}$.

Debido a lo anterior y otros retos adicionales que implica la exploración de esta cuenca, los proyectos de perforación requieren inversiones bastante altas y es común ver cronogramas de 10 - 12 meses por pozo.

Proyecto de Reacceso: Debido a la baja resolución sísmica de algunas zonas y a la incertidumbre del contacto agua-aceite (WOC), por la calidad exploratoria de los yacimientos inferiores; es posible que algunos pozos no logren los objetivos ó encuentren algunos no pronosticados. Para el primer caso, donde por la baja resolución, el pozo ha fallado el objetivo por distancia moderada; una alternativa es la construcción de reaccesos reutilizando el revestimiento intermedio. También es posible hacer reaccesos, cuando se ha interceptado la estructura objetivo por debajo del WOC, con el fin de ir hacia la parte más alta de la estructura. La figura 7 ilustra ambos casos.

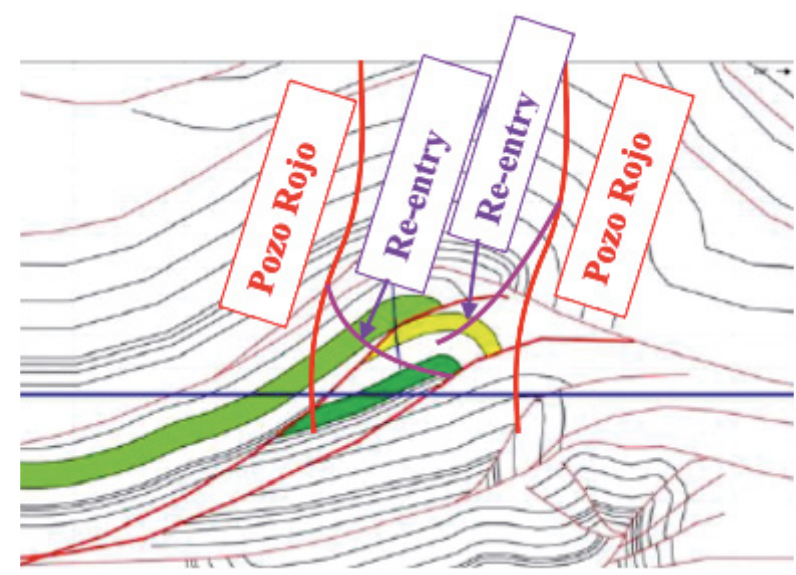

Figura 7. Casos de re-entries exploratorios: El pozo rojo ha caido debajo del WOC (Caso izquierda) y se ha hecho re-entry (Púrpura) para llegar a la zona alta de la estructura. El pozo rojo ha pasado por el frente de la estructura (Caso derecha) y se ha hecho re-entry para llegar a la estructura. 
Los aspectos de diseño relevantes, para los proyectos de reacceso en la cuenca en general, se resumen en la figura 8 y se detallarán en la siguiente sección, con el caso de estudio.

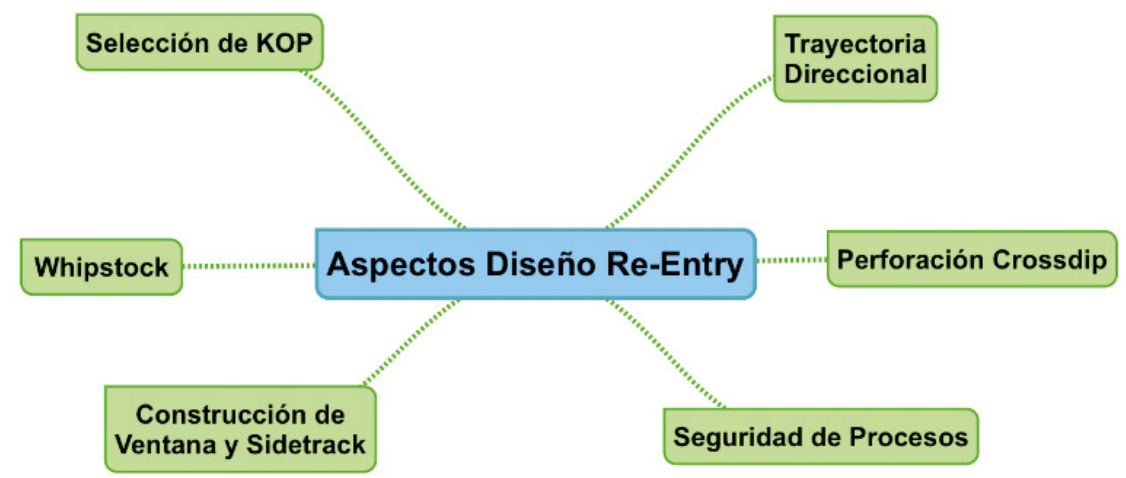

Figura 8. Aspectos relevantes del diseño de reacceso.

- Selección del punto de partida, o KOP (Kick-off point): Es el punto de inicio de la desviación del pozo original (en adelante referido como sidetrack) del pozo original. La escogencia de este punto dicta el diseño de la trayectoria para la llegada al objetivo. Para los reaccesos en la cuenca, hay varios aspectos a tener en cuenta para esta selección, que se detallarán en la siguiente sección.

- Cuchara de desviación o Whipstock: Es la herramienta que permite la desviación del pozo original. Para el caso de la experiencia de reaccesos en la cuenca, se mostrará en términos generales el diseño y algunos aspectos a tener en cuenta.

- Construcción de la ventana: Es la columna vertebral del reacceso. Esta operación tiene una gran influencia en el desempeño de la operación futura, ya que es el punto a través del cual pasarán todos los ensamblajes que construirán el hueco nuevo. Debido al alto impacto de abrasión y alta resistencia compresiva de las rocas, la perforación del nuevo hueco puede tomar decenas de ensamblajes.

- Trayectoria direccional: El diseño de la trayectoria está supeditado a los objetivos definidos, la trayectoria del hueco madre, el límite técnico de curvatura, algunas consideraciones económicas y se hace en conjunto con la selección del KOP.

- Perforación en dirección ortogonal al buzamiento (cross-dip): La dirección relativa del hueco tiene impacto en el comportamiento de estabilidad de las formaciones con presencia de planos de debilidad. Algunos aspectos del diseño del fluido de perforación y de los BHA, son críticos para mitigar el riesgo de incidentes operativos relacionados a la dirección relativa y el ángulo de ataque.

- Seguridad de procesos: Este es el corazón de las operaciones de exploración en la cuenca. La política de seguridad de procesos, que apunta hacia las personas, los procedimientos, la planta y los procesos; ha permitido llevar a cabo la campaña sin incidentes catastróficos de integridad.

\section{Aspectos del Diseño del reacceso desde el Revestimiento Intermedio}

A continuación, se mostrará en detalle algunos de los aspectos clave en el diseño de un reacceso exploratorio desde el revestimiento intermedio, ejemplificando con un caso de estudio. Se trata de un pozo exploratorio, perforado en el 2016, que cayó a la parte baja de la estructura (Debajo del WOC - contacto agua-aceite). Como alternativa de exploración a un pozo desde superficie, se concibió un reacceso desde uno de los pozos al sur del campo.

Estado mecánico. Con el fin de alcanzar el objetivo, con el mayor desplazamiento hacia el Noreste, buscando la parte alta de la estructura, se definió un KOP (a 9426 $\mathrm{ft} \mathrm{MD}$ ) en el revestimiento de 11 3/4" 65 ppf, P-110HC, tal como lo muestra la figura 9. Este revestimiento tenía un desgaste previo, resultado de la perforación del pozo original, y el cual está evidenciado en el registro ultrasónico que se muestra en la figura 10. Este era uno de los riesgos de la operación y que debía mitigarse durante la perforación del reacceso. Debía disminuirse la cantidad de rotación, optimizar la cantidad de viajes, monitorear la cantidad de metales sacados del pozo, evaluarse la condición final del revestimiento y utilizar protección activa para el desgaste (Protectores no rotativos), a partir de la simulación de fuerza lateral durante la perforación del sidetrack. 


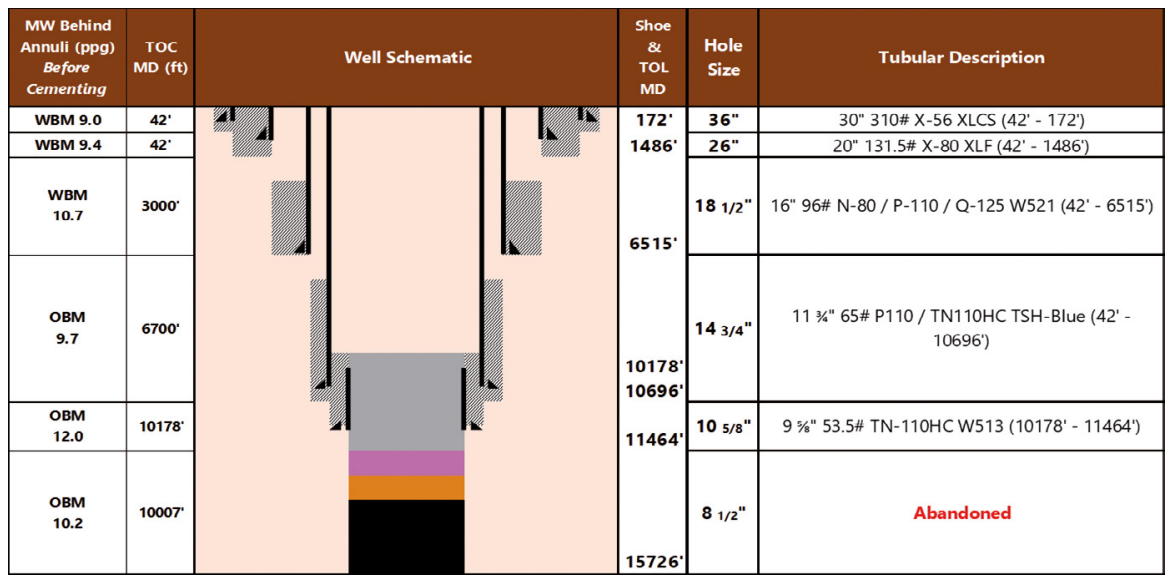

Figura 9. Estado mecánico del pozo de estudio para re-entry exploratorio.

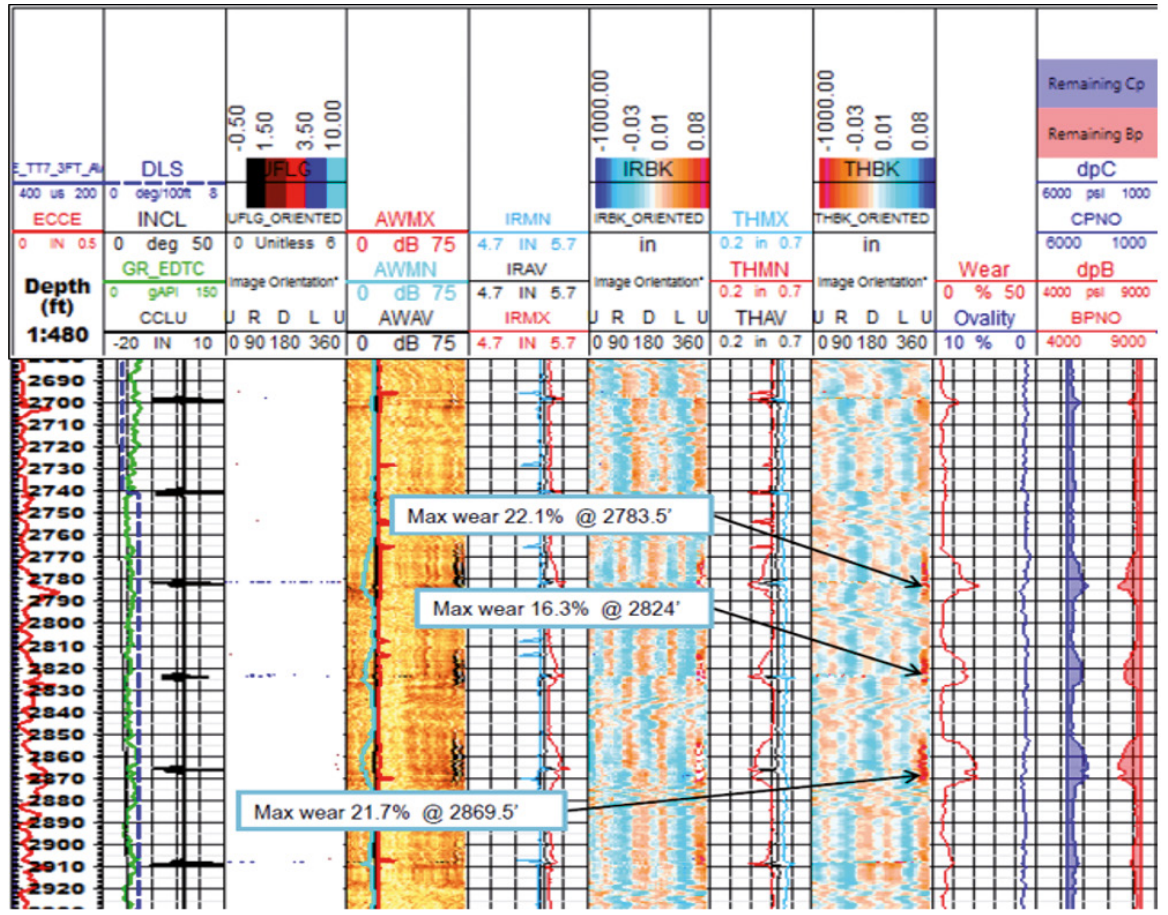

Figura 10. Registro de desgaste del revestimiento de 11 3/4" después de la perforación del pozo original.

Selección del punto de asentamiento de la cuchara de desviación. Para la selección del punto de asentamiento, se tomó en consideración, entre otras cosas:

- Máxima inclinación en la trayectoria para alcanzar el objetivo. La idea era no superar los $37^{\circ}$ de inclinación para el hoyo de 10.625 ", que se había planeado a la dirección ortogonal al buzamiento a través de una zona intercalada. Además, era necesario pasar una falla que se extiende en todo el campo, debajo de la cual se concentraba la mayor cantidad de NPT en operación de perforación, según los offset. El límite de $37^{\circ}$ estaba establecido por limpieza del hueco en un escenario de generación de derrumbes en volúmenes considerables. Cabe resaltar, que la inclinación máxima de este pozo fue $41.69^{\circ}$ debido al trabajo de sidetrack usando un motor de fondo con deflexión de $1.2^{\circ} \mathrm{y}$ broca tricónica, que excedió el desempeño esperado. No se tuvo ningún problema operativo relacionado a la limpieza del hoyo.

- Competencia de la zona de asentamiento de la cuchara. Se escogió una formación con alta resistencia compresiva que haya tenido un tiempo 
de exposición relativamente bajo, con el fin de tener alta probabilidad de estar en zona en calibre. Las ventajas de esto:

- Menor probabilidad de lavado de la formación y por tanto menor espacio anular.

- Menor probabilidad de fallar el sidetrack y caer en el hueco original.

- Mayor posibilidad de tener mejor calidad del cemento detrás del revestimiento.

- Menor probabilidad de vibración de la sarta de molienda de la ventana.
- Estado del cemento detrás del revestimiento. Una mala condición de cemento detrás del punto de KOP aumenta el riesgo de daño prematuro del moledor usado para abrir la ventana, debido a la condición dinámica de la sarta (Vibración) y por tanto hace necesario la corrida de rectificación. También aumenta el riesgo de fallar el sidetrack, si no se hace una selección adecuada del ensamblaje para completar el sidetrack incluyendo la broca. La figura 11 muestra el número de viajes requeridos para la construcción de la ventana en revestimientos intermedios, con calidad de cemento regular.
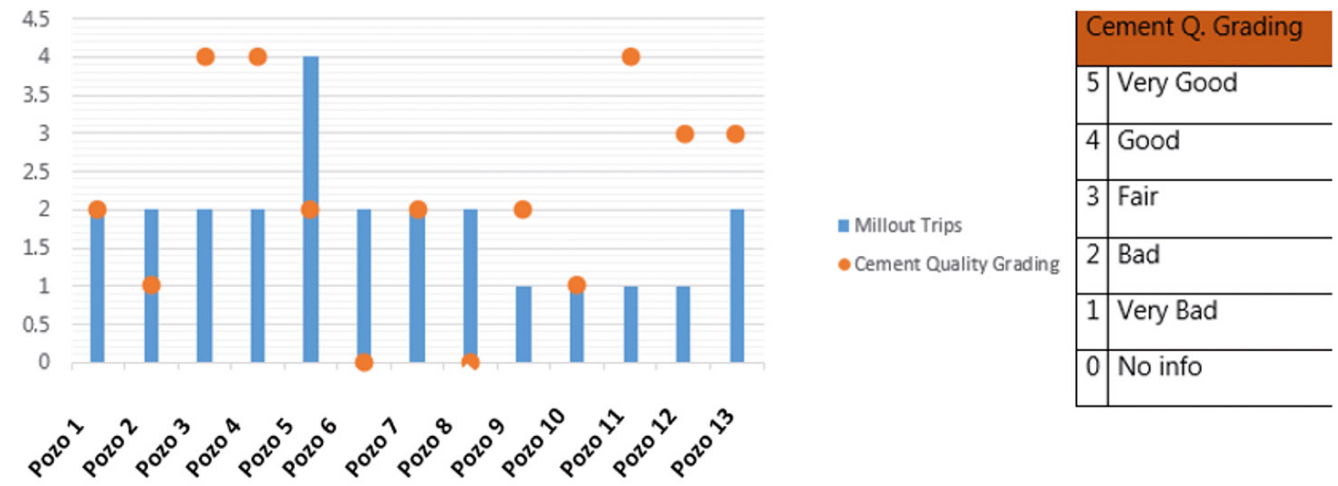

Figura 11. Número de viajes para apertura de la ventana según condición del cemento.

Como consecuencia a las razones mencionadas, se escogió el asentamiento de la cuchara en la zona que se muestra en la figura 12, orientado hacia el lado alto. La orientación hacia el lado bajo contempla riesgos adicionales. En este caso, el lado bajo tenía un canal sin cemento apreciable en el registro ultrasónico, además de la complejidad geométrica del paso por la ventana para los BHA de perforación.

\section{Milling de ventana en revestimiento intermedio.}

Para la molienda de la ventana, es necesario hacer un diseño detallado de:

- Diseño del moledor. Debe ser lo suficientemente robusto para la molienda del metal del revestimiento intermedio con grado P110HC. Es importante resaltar que la dureza del revestimiento es dependiente del grado del metal. Para el caso del revestimiento $110-\mathrm{HC}$, a diferencia del grado $\mathrm{P}-110$, tiene una mayor resistencia al colapso. Teóricamente, esta puede darse con la revisión de calidad del espesor de pared del tubular y certificando que podría soportar mayor carga de colapso. No obstante, otra manera de hacerlo, es construyendo tubulares con una resistencia mínima antes de cedencia (conocido en inglés como minimum yield stress) mayor. Por ejemplo, un tubo Q-125 tiene una capacidad de resistencia al colapso comparable con un tubo P-110 HC para este tamaño. Es común ver lotes de tubos P-110HC con MYS del orden de los 125 - 135K psi. La dureza medida en un lote de inventario con el mismo grado, estuvo en el orden de los 240 - 270 brinell.

El moledor debe ser también lo suficientemente robusto para encarar la formación detrás del revestimiento. En este caso, se trata de una arenisca abrasiva con alta resistencia compresiva (>20K psi). La perforación del bolsillo (conocido en inglés como rat hole) en la construcción de la ventana, debe ser suficiente para sacar el moledor secundario al hueco abierto y dar espacio al BHA que completará el sidetrack. La figura 13 muestra el diseño del moledor principal usado para la molienda de la ventana. 


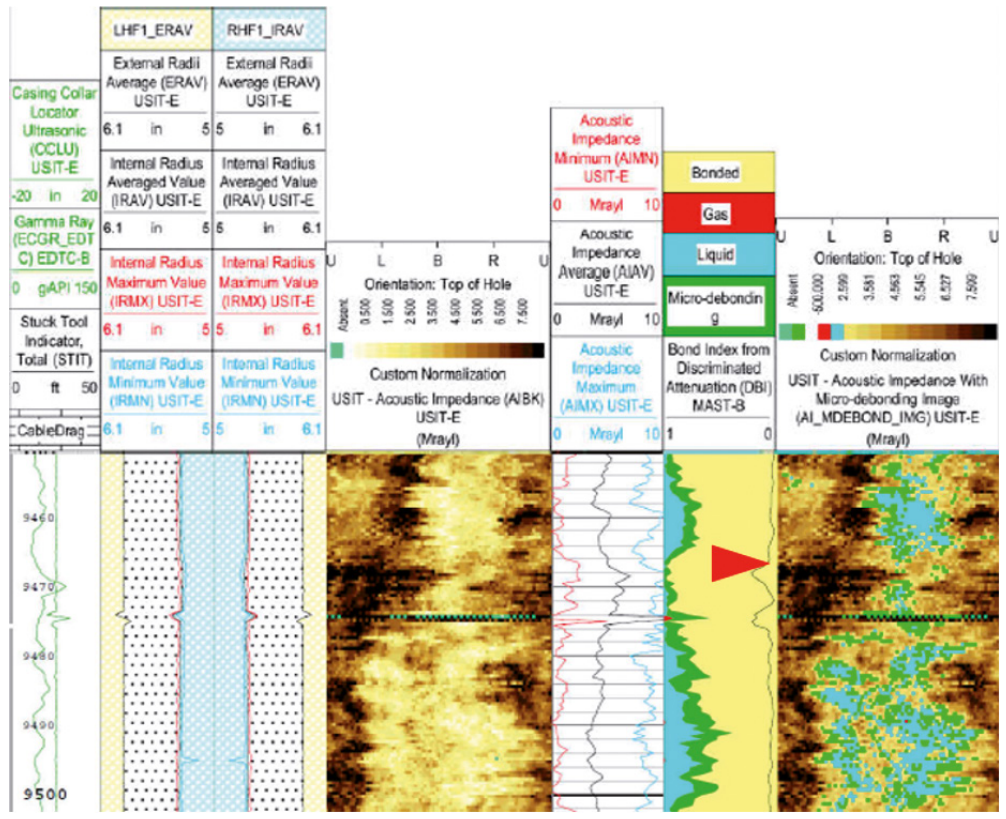

Figura 12. Zona escogida para el asentamiento de la cuchara, en la cara alta del hueco.

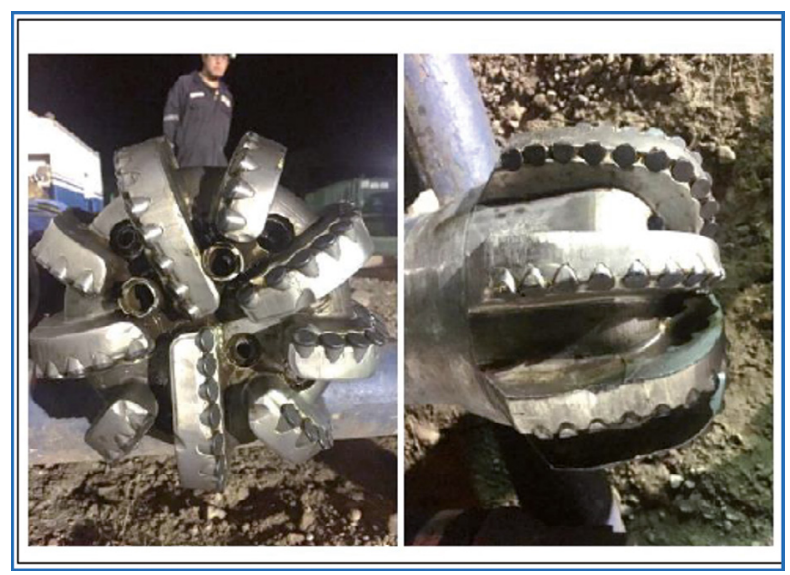

Figura 13. Moledor PDC usado en la apertura de la ventana.

- Diseño de la cuchara. Tal como se mencionó, es la columna vertebral del reacceso. La geometría de la ventana es función del diseño de la rampa. El material de la rampa debe ser lo suficientemente robusto, para soportar el trabajo que se hará para construir el lateral, que en el caso de la cuenca sub-andina, implica meses. Para el caso del pozo de estudio, se escogió el sistema trackmaster de Schlumberger, cuya rampa tiene una geometría de etapas. La sección de la media rampa tiene la mayor inclinación y permite la flexión necesaria para apuntar la sarta hacia el hueco nuevo. La figura 14 muestra un esquema del sistema usado.

- Mapa de parámetros para la construcción de la ventana. Considerando la condición del cemento, el diseño del mapa de parámetros, con base en la dinámica de sarta, es clave para mitigar el riesgo de daño prematuro del moledor. La figura 14 muestra también la simulación de dinámica de sarta para este caso. Puede observarse la limitación del mapa de parámetros (velocidad de rotación y peso sobre la broca, RPM/WOB) dada la condición de vibración lateral y torsional.

Adicional a esto, durante la apertura de la ventana y durante el paso por la ventana, el ensamblaje o la tubería son sometidos a un esfuerzo de flexión alto y debido a la rotación desde superficie, las conexiones cercanas a esta zona de alto esfuerzo de flexión, se someten a ciclos de esfuerzos. La figura 15 ilustra este concepto. Vale la pena detenerse para detallar un poco el análisis de momento de flexión que se realizó para el pozo de estudio. Al respecto del esfuerzo de flexión y los esfuerzos cíclicos:

- Los esfuerzos de flexión resultan en un esfuerzo de tensión y compresión para los extremos opuestos del tubular sometido al esfuerzo, en la curvatura.

- El esfuerzo de tensión se intensifica en la zona cercana a las juntas de tubería cuando el tubular se somete a tensión y se intensifica en el cuerpo del tubo cuando el tubular se somete a compresión. 


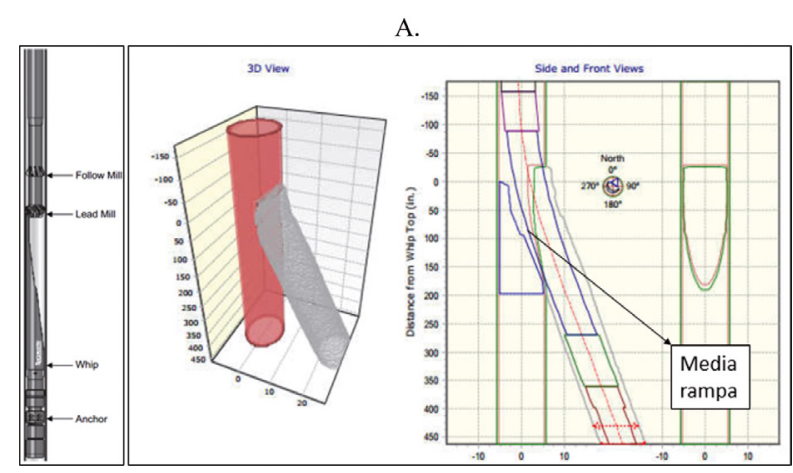

B.

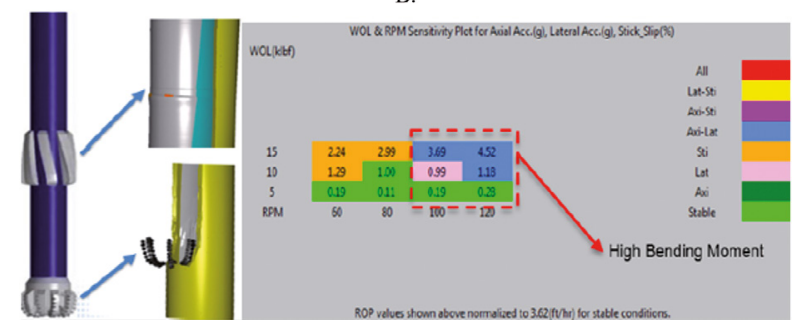

Figura 14. A. Esquema de la ventana y la cuchara usado. B. Simulación de dinámica de sarta Media-rampa.

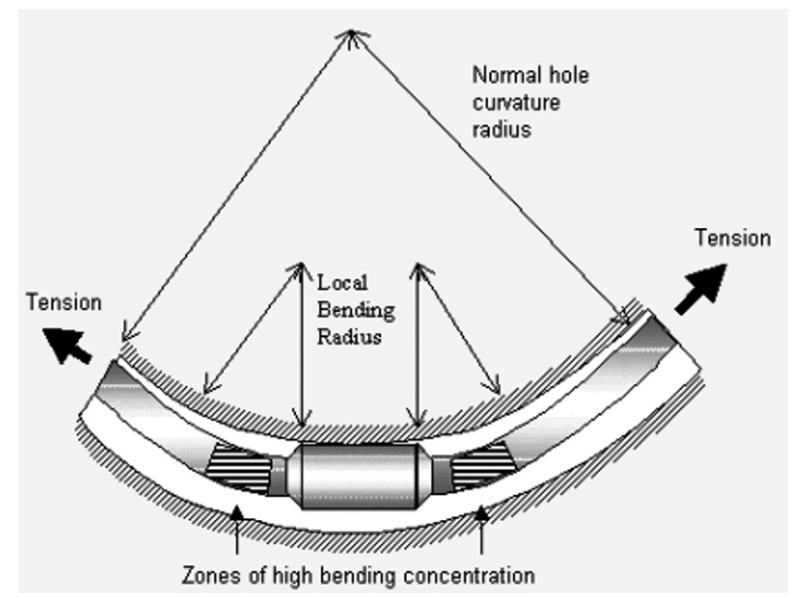

Figura 15. Ilustración del momento de flexión en el cuerpo del tubo y las juntas de la tubería.

- Los autores coinciden en que un tubular sometido a un esfuerzo de flexión, tiene probabilidad de falla por fatiga. (API, 1981; Lubinski, 1961; Shokir, 2004; Stelzer, 2007; Vaisberg et al., 2002).

- Un análisis de fallas de tubulares, demuestra que la mayoría de los eventos (65\%) ocurre por fatiga. Tan sólo un $8 \%$ ocurre por exceso de esfuerzo puntual (Vaisberg et al., 2002). Vale la pena aclarar, que flexión y pandeo son fenómenos distintos. En este caso no se tenía condición de pandeo, dado que la tubería era robusta ( 5 " y 5 7/8" S-135), que no se superaba el límite de peso aplicado para pandeo sinusoidal, y que la fuerza de fricción adicional (dada la fuerza lateral) en ambiente de alto DLS, ayuda en dar soporte para evitar el pandeo. Sin embargo, si se tenía alto DLS y por tanto flexión de la tubería.

- La falla por fatiga en un tubular sometido a esfuerzo de flexión no se da por el esfuerzo en sí, sino por el sometimiento a esfuerzos cíclicos. La dinámica más importante que causa esos esfuerzos cíclicos es la rotación. Algunos autores también mencionan la vibración. Sin embargo ese punto no se considera en este documento. La rotación, en especial a ROP bajas, someten un mismo punto a variaciones de tensión y compresión que llevan a la fatiga. Ese fenómeno, según la literatura, se da gracias a fracturas que aparecen por dislocaciones de la estructura del metal, como la que se muestra en la figura 16. La tensión adicional es un factor que amplifica las fracturas y reduce la vida útil del tubular. Este fenómeno de reducción del máximo esfuerzo de flexión se ha computado en una relación empírica propuesta por Goodman (Lubinski, 1961).

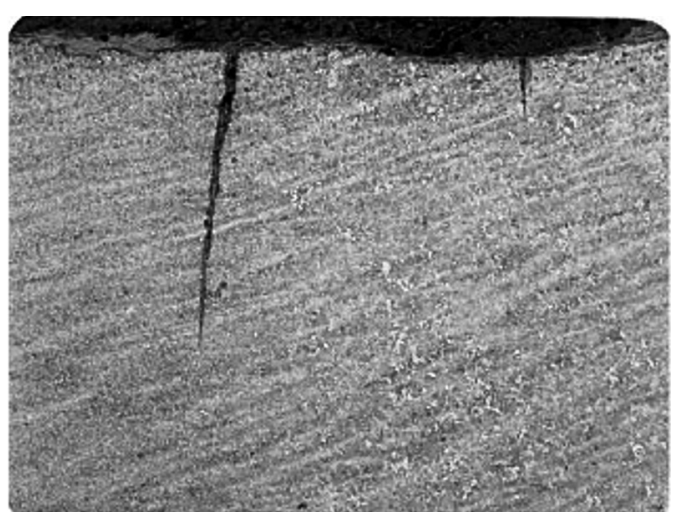

Figura 16. Fractura en el metal producida por fatiga.

- El análisis de fatiga es afectado por la geometría del DLS (Dogleg severity). i.e. Si la curvatura es abrupta o gradual, ya que esto influye en el contacto del tubular con la superficie de la formación/casing.

- Los diagramas N-S se obtienen de pruebas llevadas a cabo con materiales que son sometidos a cargas cíclicas hasta la falla. A partir de estas, puede determinarse para un dado esfuerzo de flexión al que es sometido un tubular, cuál es la máxima cantidad de ciclos tensión-compresión que podrían permitirse antes de que el material falle por fatiga. 
- La literatura propone entonces 2 cálculos de interés:

$\therefore$ Máximo dogleg permitido para evitar fatiga del material.

i. Se calcula el máximo bending stress para el grado de acero del tubular en análisis.

ii. Se calcula el máximo dogleg permisible para no tener eventos de fatiga.

$\because$ Máximas revoluciones a las que puede someterse un tubular para un determinado dogleg.

i. Se calcula el esfuerzo de flexión, dadas las condiciones a las que está sometido el tubular de tensión / compresión y dogleg.

ii. Se calcula el máximo número de ciclos según diagrama N-S para el grado de metal del tubular.

iii.Se calcula el número acumulado máximo de revoluciones que corresponden a ese número de ciclos.

Morgan y Roblin encontraron una buena correlación entre la resistencia del tubular y el límite de resistencia a la fatiga. A partir de su estudio, se desarrolló una gráfica directa del máximo esfuerzo de flexión contra revoluciones, que puede usarse acumulativamente. No obstante, el uso de esta gráfica requiere el conocimiento de la trazabilidad completa de los tubulares. i.e. La historia completa de su uso. En muchas ocasiones, sobre todo para tubería rentada, esta información no está disponible (Stelzer, 2007).

- La literatura propone también un procedimiento de inspección para tubulares que han sometido a esfuerzos de flexión y cíclicos. Este no se menciona en este documento. Algunos factores que se tienen en cuenta:

- Ambiente no corrosivo.

- Debe tenerse en cuenta la inexactitud de las desviaciones (conocido por su término en inglés como survey), para el cálculo del máximo DLS. Para esto, en la literatura se propone un factor de corrección.

- Las marcas de cuñas pueden reducir el número de ciclos del diagrama N-S, al igual que la máxima tensión para determinado esfuerzo de flexión en el diagrama de Goodman.
- Si el tubular está en contacto con la pared del hueco o del casing, donde se encuentre el dogleg, esto genera un desgaste en el tubo. Si se trata por ejemplo de un tubular sometido a esfuerzo de compresión, donde el esfuerzo de flexión se intensifica en el cuerpo del tubo, entonces el desgaste generado por el contacto puede reducir el número de ciclos del diagrama N-S, al igual que la máxima tensión para dado esfuerzo de flexión en el diagrama de Goodman.

- Los software de ingeniería de perforación, tienen módulos para el análisis de fatiga de los tubulares. En el caso de DSWE (propiedad de Landmark), se calcula el esfuerzo de flexión y pandeo para determinadas condiciones especificadas de RPM, WOB, ROP. Además, se calcula el límite de resistencia a la fatiga, considerando lo siguiente: «The number of cycles for the fatigue endurance limit is approximately taken at 107 rotations. This is the level of cyclic stress beyond which the material is immune to fatigue failure». Luego se dividen ambos cálculos y se obtiene la razón de fatiga, que debe ser menor a 1 para todos los BHA. Cabe aclarar que este límite no es un caso particular, sino la premisa sobre la cual está construida el simulador.

- Debido a que la fatiga se da por aparición y posterior propagación de micro-fracturas, la literatura recomienda un proceso de inspección más riguroso a herramientas sometidas a esfuerzos cíclicos en cuanto a tipo y frecuencia, para la detección de esas fracturas. Para este caso particular, se recomienda inspección con ultrasonido y electromagnetismo (UT/EMI), enfatizando en las zonas más susceptibles a fatiga en tubos o herramientas en tensión: Los tool joints. Para frecuencia, se habla de 150 hrs en el caso más conservado (Vaisberg et al., 2002).

Para el pozo en estudio, durante la apertura de la ventana, el momento de flexión esperado se magnificaba detrás del moledor, tal como se muestra en la figura 17. Además de esto, durante la perforación del sidetrack, todas las herramientas pasando por la ventana, estarían sometidas a esfuerzos cíclicos, dado el alto DLS. 


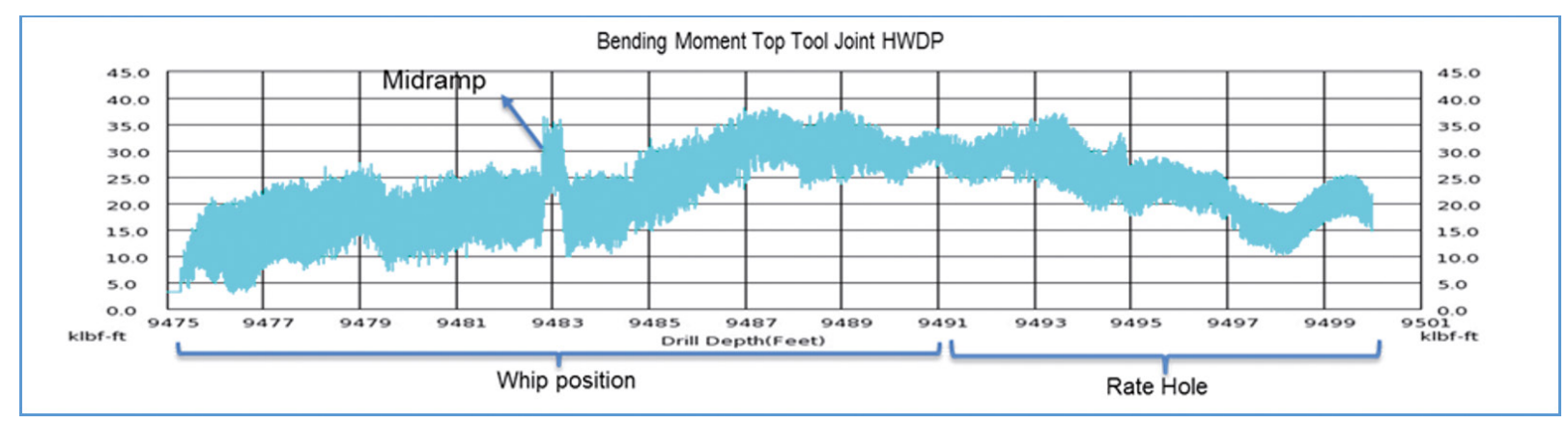

Figura 17. Momento de flexión durante la perforación de la ventana para la herramienta detrás del moledor.

Dada la situación anterior, los siguientes puntos fueron acordados para la perforación del reacceso:

- Uso de una junta de HWDP detrás del moledor, dado que esta es la herramienta más resistente a esfuerzos de fatiga.

- En lo posible, no hacer repaso en reversa (backreaming) de las paradas ó máximo 1 junta.

- Backreaming al final de cada sección perforada por cada BHA, y bombeo (pumping out) del resto de la sección hasta estar por encima del tope de la cuchara.

- De ser posible, correr los ensamblajes sólo lavando hacia abajo (wahing down) hasta 1 parada antes de la profundidad del fondo.

- Evitar en lo posible altas RPM. Si es posible mantener por debajo de $80 \mathrm{rpm}$. A mayor RPM la sarta se comporta de manera más rígida y fatiga más los puntos posicionados en alto DLS.

- Se inspeccionan las herramientas pasando por alto DLS con una frecuencia máxima de 150 horas de rotación. Además, se cambiarían estas herramientas en cada viaje.

- Limpieza del metal removido. Debido a la alta gravedad específica del metal, un riesgo contemplado en la operación de apertura de la ventana, es la imposibilidad de limpieza de los metales cortados del revestimiento. Además, si el fluido pudiera llevar los recortes a superficie, también existe el riesgo de no poder removerlos de él. Esto podría resultar en: Ineficiencia de perforación, daño de herramientas ó incluso, pega de tubería.

Como control a este punto, se implementó el uso de magnetos de alto poder ( 8 en total), dispersos en el bolsillo (o caja de distribución) de las zarandas y el canal de retorno a los tanques intermedios. Vale la pena resaltar que para el trabajo de molienda, se alineó el sistema de superficie en circuito corto, para evitar la contaminación del fluido.

Además, se bombeó un tren de píldoras viscosas cada $2 \mathrm{ft}$ perforados de la ventana para la remoción del metal. Cada tren de píldora consistía en un bache de baja reología, seguido por un bache viscosopesado con el fin de generar turbulencia en fondo y capacidad de levante.

Por último, se limpió el arreglo de BOP (Blowout preventors) usando una herramienta de lavado (flushing) con cierta frecuencia.

Muchos de estos puntos, han sido recolectados en los trabajos de reacceso a nivel mundial (Ugochukwu et al., 2016).

Trayectoria direccional exigente en hueco de $\mathbf{1 0 . 6 2 5}$ in. Posterior a la apertura de la ventana, se completó el sidetrack usando un ensamblaje con motor (Ultra LS, Bent: $1.2^{\circ}$ ) y Broca tricónica. La escogencia de esta broca, se dio con las siguientes premisas:

- Geometría de cortadores (Esféricos) del gauge con baja probabilidad de generación de torque reactivo (Mejor control de la orientación del motor, en adelante referido por su término en inglés: Tool face).

- Broca con la mejor capacidad de direccionamiento, para asegurar el sidetrack.

- Broca con insertos de carburo de tungsteno, con la capacidad de perforar la formación con alta resistencia compresiva.

- Broca con protección en la pata suficiente para asegurar el mantenimiento del calibre en una formación abrasiva.

- Broca con cortadores resistentes en el borde 
de la broca (conocido por su término en inglés como Hill), para soportar la carga lateral impresa por la deflexión del motor.

- Sistema de rodamientos resistentes para asegurar integridad de los conos en un ambiente de altas cargas durante la perforación.

La figura 18 muestra la broca escogida.
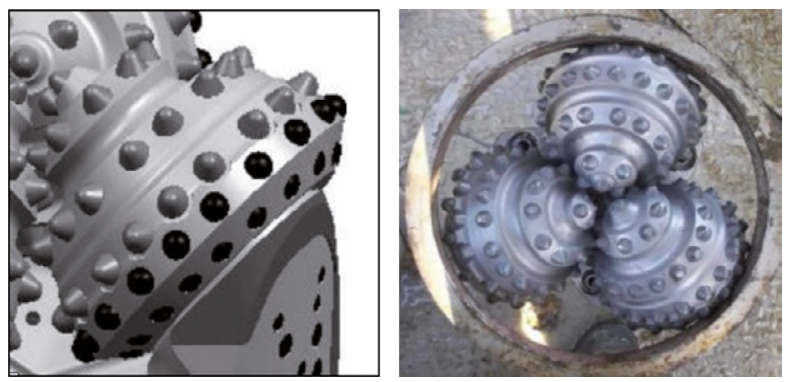

Figura 18. Broca escogida para completar y asegurar el sidetrack del hueco original.

La confirmación de sidetrack se dio con el monitoreo de las muestras y de la inclinación (lecturas de azimut no se tenían debido a la interferencia magnética con el revestimiento anterior), logrando mantener el Tool face de la herramienta estable en el rango de $125^{\circ}$ $135^{\circ}$ (bajo torque reactivo) y una curvatura máxima de $10.89^{\circ} / 100 \mathrm{ft}$.
Se continuó perforando el hueco de 10 5/8" con ensamblajes de RSS (Rotary Stereable system) + MWD $\&$ Copilot + broca PDC a través de la formación abrasiva siguiendo un mapa de parámetros simulados para sacar el mayor desempeño posible y mantener la sarta estable con bajas vibraciones. Se tomó surveys cada $45 \mathrm{ft}$ y se usó la lectura continua de inclinación y azimut, con el ánimo de monitorear la separación centro a centro con el pozo original. Se midió además el momento de flexión (BM/BMTF) para analizar tendencias de la formación y tomar decisiones en avanzada en la configuración de la herramienta direccional, evitando generar alta tortuosidad y asegurar la corrida del liner 9 $5 / 8$ ". Vale la pena aclarar, que dado el diseño del pozo, la corrida del liner de $95 / 8$ " en hueco de $105 / 8$ " es un reto en términos de posibles restricciones geométricas, debido al tamaño tan reducido del anular. Adicional, debido a las fallas de la zona, es posible encontrar cambios de tendencia de formación que requerirían mayor resolución de surveys (Mayor frecuencia) para ser detectadas oportunamente antes de generar extratortuosidad. Aquí es precisamente donde entra en juego el monitoreo del BM/BMTF. La figura 19 muestra un intervalo de cambio brusco de tendencia, detectado mediante el monitoreo de BM/BMTF, que permitió la configuración oportuna del RSS para dar respuesta y disminuir la tortuosidad del hueco.

\begin{tabular}{|c|}
\hline $\begin{array}{l}\text { Evidenced strong } \\
\text { tendency to drop } \\
\text { inclination at entrance to } \\
\text { Guadalupe Sh. Increased } \\
\text { BF to } 48 \% \text { to control } \\
\text { trend and avoid severe } \\
\text { tortuosity. }\end{array}$ \\
\hline $\begin{array}{l}\text { BMTF between } 120- \\
140^{\circ} \text { showed turning to } \\
\text { the right job and drop } \\
\text { caused by tendency }\end{array}$ \\
\hline $\begin{array}{l}\text { WF was increased on } \\
\text { time up to } 72 \% \text { to } \\
\text { maintain proper turning } \\
\text { rate of } 6.5-7.0^{\circ} / 100 \mathrm{ft}\end{array}$ \\
\hline
\end{tabular}

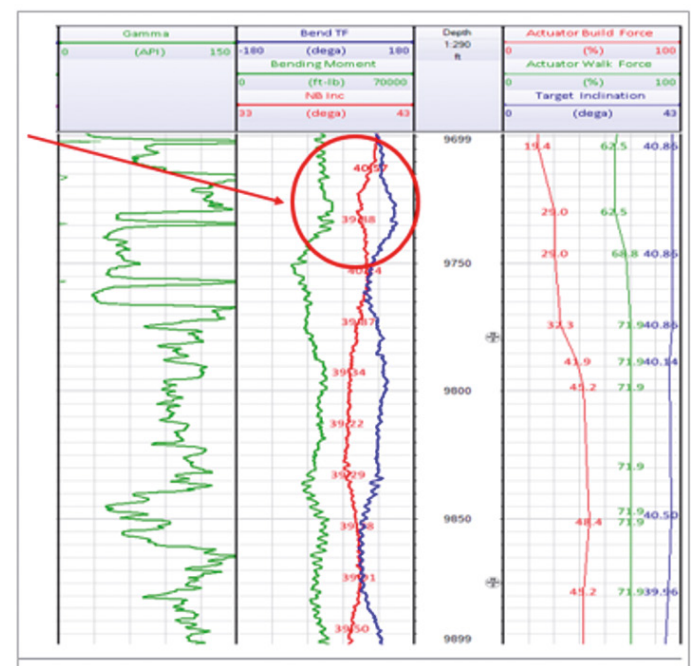

Figura 19. Monitoreo del BM/BMTF en zona de cambio de tendencia.

Posteriormente, se continuó la trayectoria a través de una zona intercalada, girando hacia la dirección ortogonal al buzamiento a una tasa de 6.0 a $7.6^{\circ} / 100 \mathrm{ft}$. La máxima inclinación alcanzada en esta sección fue
41.69 $9^{\circ}$. En este intervalo se tomaron lecturas de Gamma Ray Azimutal para leer el buzamiento y dirección de las capas y de esta manera poder correlacionar el modelo estructural, ajustándolo en caso de ser necesario. 
En la sección de 8 1/2” se perforo con lodo nuevo limpio de tipo yacimiento (bajo contenido de sólidos) con una densidad entre 9.3 a 10.4 ppg a través de una zona

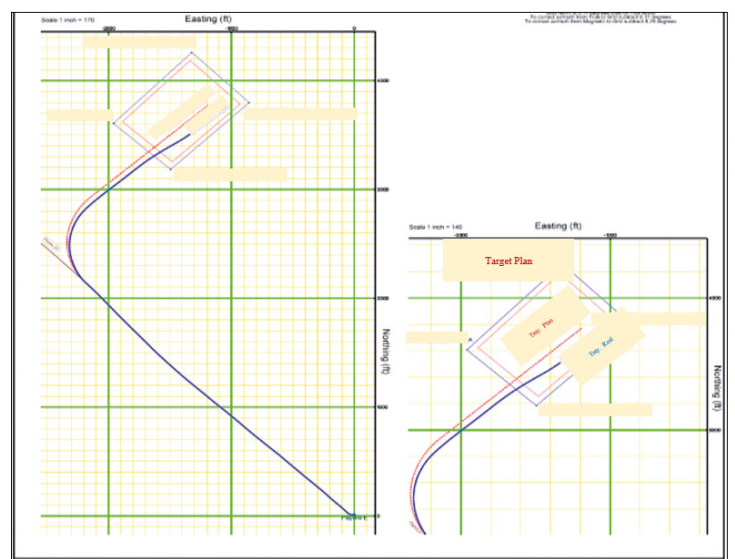

Figura 20. Trayectoria direccional del pozo de estudio.

La operación de molienda, perforación y orientación direccional a la dirección ortogonal al buzamiento fue un éxito luego de la corrida de doce ensamblajes de fondo (BHAs) basado en los siguientes criterios y estrategias:

- Adecuado diseño de la broca tricónica en términos de protección lateral y bajo torque reactivo.

- Uso de la herramienta Copilot para prevención de la generación de tortuosidad en zona fallada.

- Estrategia adecuada para manejar la interferencia magnética con el hueco cercano.

- Bombeo de píldoras para limpieza de viruta de Metal.

- Uso de brocas de última tecnología para roca dura y abrasiva.

- Mantener la configuración entre ensamblajes reduciendo el riesgo de restricciones geométricas.

- Corrida de la herramienta Gamma Ray Azimutal para un análisis estructural

- Adecuado manejo de la estrategia de sello del lodo carbonera (10 5/8") y yacimiento (8 1/2").

Perforación de zona fallada en dirección ortogonal al buzamiento. La perforación en dirección cross-dip (X-Dip) ha sido teórica e históricamente un reto desde el punto de vista de estabilidad, por ser la menos una de las menos preferentes. No obstante, algunos temas del diseño, han permitido que pozos perforados en dirección cross-dip, no tengan problemas operativos en absoluto. Adicional a esto, es necesario combinar el análisis con el ángulo de ataque y la tendencia de derrumbes en pozos de correlación, en un análisis combinado de severidad de trayectoria. Para el caso del fallada. La figura 20 muestra la sección vertical y la vista en planta para la trayectoria descrita.

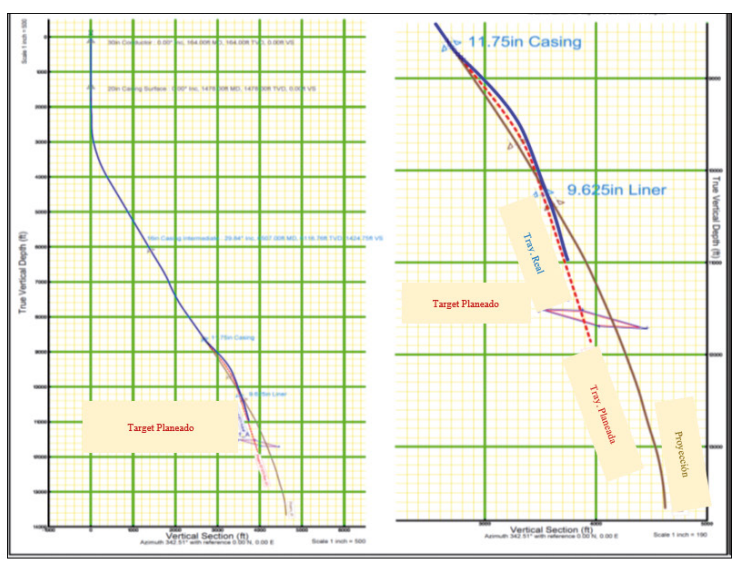

pozo de estudio, tal como puede observarse en la figura 20, la mayor parte del pozo, incluyendo el paso por la falla de la que se ha hablado, se hace en dirección crossdip. El concepto de la perforación cross-dip se ilustra en la figura 21. Consiste básicamente en perforar de manera transversal al buzamiento de la capa.

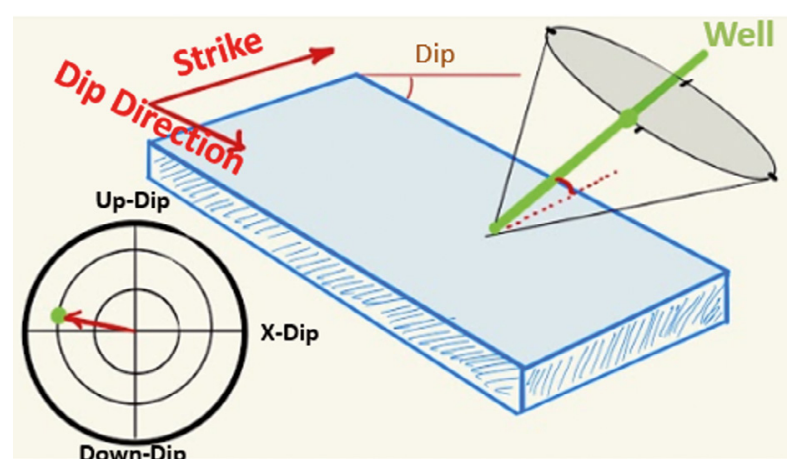

Figura 21. Dirección relativa del pozo para el análisis de severidad de trayectoria.

La corrida de un modelo probabilístico, permitió el diseño del peso de lodo. Adicional a esto, se utilizó una distribución de tamaño de partícula (PSD) con el uso de nano-partículas, para el puenteo de gargantas porales y planos de debilidad. La figura 22 muestra el análisis de trayectoria en términos de geomecánica para el pozo de estudio. El diagrama polar, muestra la severidad desde el punto de vista del ángulo de ataque. En la escala de color del diagrama polar, el extremo azul representa la zona de menor riesgo de inestabilidad de las paredes del pozo. El extremo rojo, representa la zona de mayor riesgo. 
Desde el punto de vista de fluidos, además del PSD con carbonato de calcio de distinta granulometría, se mantuvo una concentración moderada de asfaltos y grafitos, para ayudar en la estabilización de la pared del hueco. Como complemento de la estrategia de peso de lodo, se tuvo un plan detallado de concentraciones, usado para atravesar la falla que se ha mencionado, incluyendo el uso de nanopartículas poliméricas. Varias aplicaciones se han realizado a nivel mundial, con el objetivo de estabilizar zonas arcillosas con fisilidad (Contreras et al., 2014; Hoelscher et al., 2013).
Como resultado, la máxima tasa de producción de derrumbes fue de 1.6 barriles por hora, con un $90 \%$ de morfología tabular (Producto de planos de fisilidad). La figura 23 muestra el monitoreo de estabilidad de la sección de 10.625 ", perforada con la estrategia de lodo descrita. Algunos puntos a resaltar:

- No se observa morfología de re-trabajados que pudiesen indicar falla de limpieza del hueco.

- No se observa exceso de producción de derrumbes debajo de la falla, mostrando una estrategia de sello y estabilización efectiva.

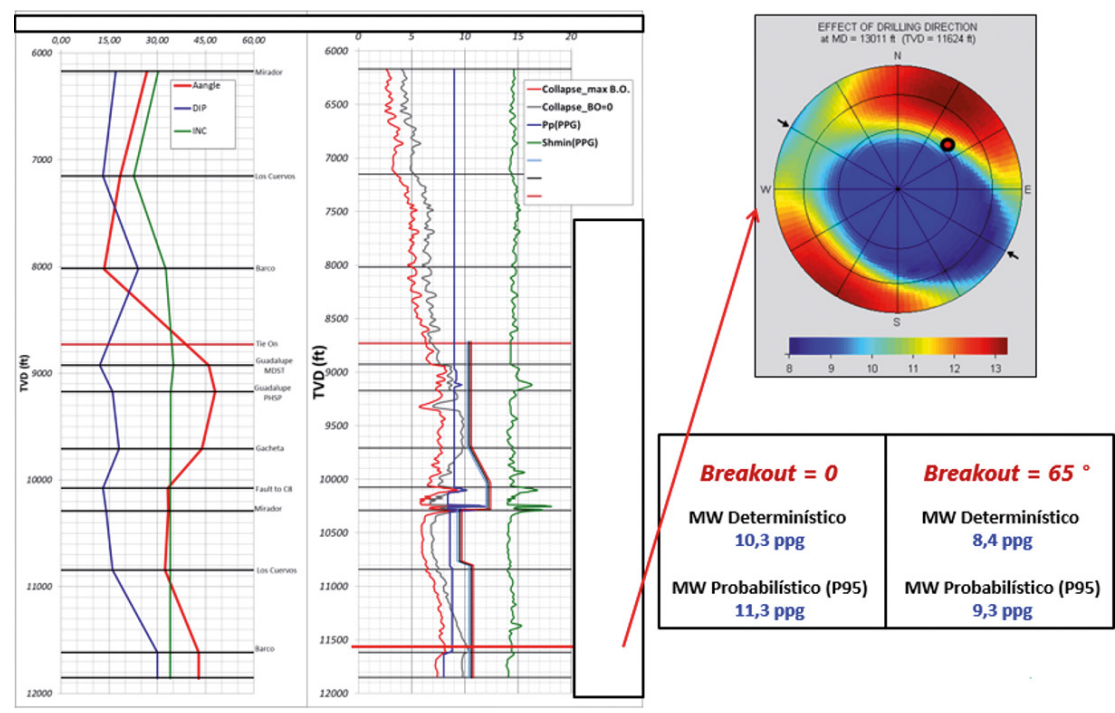

Figura 22. Análisis de severidad de trayectoria y probabilístico para pesos de lodo requeridos.

En la figura 23, el diagrama de color azul simboliza el volumen de derrumbes producido por hora, en el eje vertical izquierdo. La línea roja delgada, simboliza la altura del bloque, medido en el eje vertical derecho. La tercera gráfica (Color verde), representa la morfología en porcentaje de la muestra de derrumbes. Como puede observarse, la mayor cantidad de derrumbes eran de morfología tabular y blocosa.

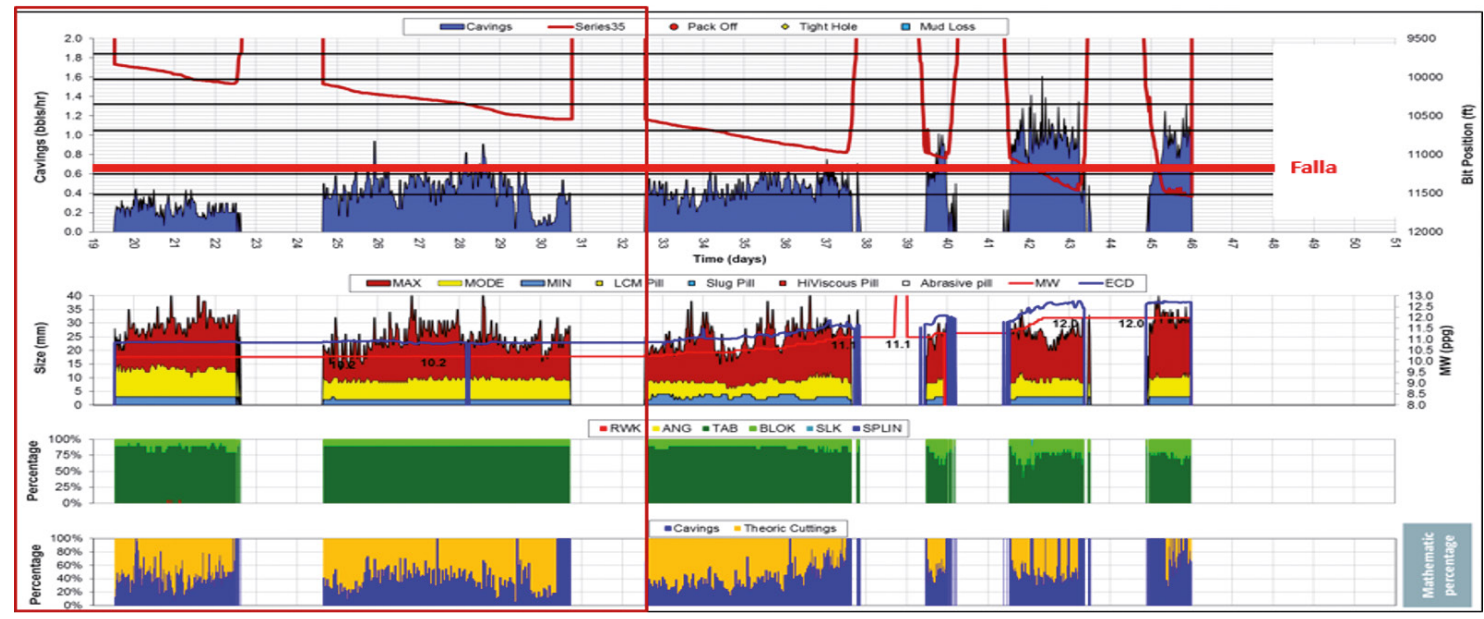

Figura 23. Monitoreo de estabilidad en sección de 10.625" del pozo de estudio. 
Retos de seguridad de procesos. El proyecto de estudio, no solo se aseguró desde la planeación y un diseño desde el punto de vista de ingeniería impecable, sino que este aseguramiento también se llevó a cabo desde el punto de vista operativo y de seguridad de procesos siguiendo un protocolo dedicado y enfocado en el aseguramiento de equipos y herramientas de la siguiente manera:

- Taladro: de la mano con el contratista de perforación y tras entender que el taladro que realizaría el proyecto era un equipo almacenado por 5 años debido al período de crisis en la industria, se tomaron las siguientes acciones:

- Se realizó trabajo de pre-comisionamiento en dos sesiones anticipadas al movimiento del taladro, donde se identificaron y acordaron temas relacionados a mantenimiento, pruebas y estados de todos los componentes del taladro. En otras palabras, el equipo de integridad de la compañía operadora se hizo partícipe del programa de mantenimiento e integridad de la compañía del taladro.

Vale la pena mencionar, que un taladro que ha sido almacenado por tanto tiempo, requiere un procedimiento de puesta a punto especial. Durante las sesiones de precomisionamiento se identificaron una buena cantidad de empaques, componentes eléctricos, válvulas, líneas hidráulicas y componentes con elastómeros, habían sufrido degradación y debían ser reemplazados. Lo anterior, se realizó en integración con la compañía operadora, aprovechando la experiencia en equipos de la planta de tratamiento y recolección de crudo y gas (Facilidades de superficie).

- Una vez movilizado y armado el taladro se realizó una sesión formal de comisionamiento, donde se probó cada sistema del taladro, sometiendo el mismo a las máximas cargas posibles que permitieran identificar fallas en equipos, tanto mecánicas, eléctricas, electrónicas o de comunicación.

- Personal: En conjunto con el contratista del taladro, se lideró un programa robusto de capacitación de personal para cargos críticos y no críticos, que llevaran al mejor desempeño y conocimiento del taladro y sus equipos. En la fases preliminares de movilización, arme y operación se contó con un grupo de entrenadores expertos de la compañía contratista del taladro que permitió adiestrar en operación del taladro al personal crítico y fortalecer sus debilidades operativas. En este factor, la variable social es interviniente y debe ser tomada en cuenta en el análisis de riesgo operativo del proyecto.

- Proveedores de servicios: La compañía operadora, a través de sus líderes de integridad, seguridad de procesos y expertos de cada línea, ejecutó auditorías a cada proveedor que prestaría sus servicios en pozo. En estas auditorías se realizaron verificaciones de estándares, procedimientos, mantenimiento y trazabilidad de equipos, personal y talleres, además de hojas técnicas de mantenimientos, procedimientos de ensamblaje de herramientas y conocimiento equipos. De estas auditorías se abrieron planes de acciones con seguimiento, las cuales se auditaron con visitas de confirmación.

La figura 24, hace un resumen de los focos de seguridad de procesos, que permitieron un tiempo no productivo record en el campo.

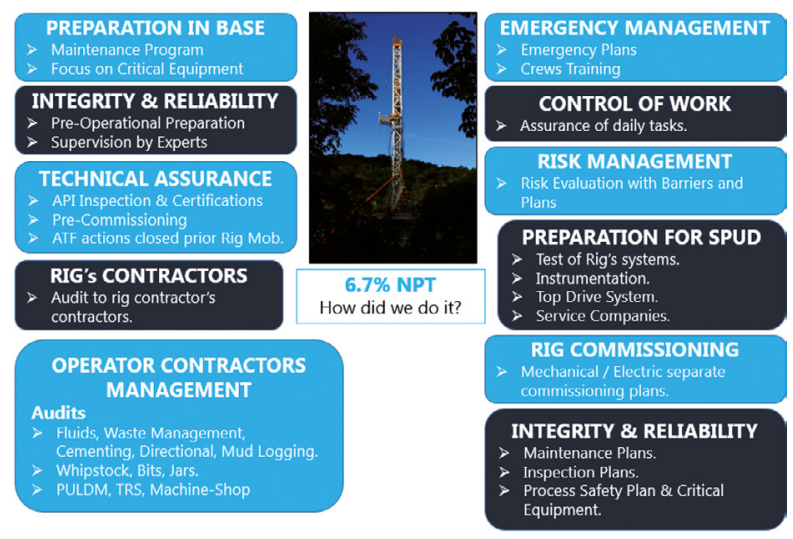

Figura 24. Puntos focales del aseguramiento de seguridad de procesos.

\section{Aplicación y Análisis de Resultados}

El pozo de estudio fue concebido con una trayectoria direccional exigente con un perfil tipo $\mathrm{S}$, divido en dos secciones de hueco.

Previo a la corrida de la cuchara de desviación, se realizó la corrida de un ensamblaje de calibración (Con el uso de raspadores) con el fin de simular la rigidez del ensamblaje del Whipstock y verificar acceso. También se bajó este ensamblaje para limpiar la zona de asentamiento del whipstock y verificar la condición del lodo base aceite (MW: $10.2 \mathrm{ppg}$ ), teniendo en cuenta que era un pozo suspendido hacía un año y siete meses. 
El fluido en el pozo suspendido, consistía en un lodo base aceite (Tipo emulsión inversa) de 10.2 ppg tipo yacimiento (Libre de asfaltos, grafitos y barita). En promedio, contenía $79 \mathrm{ppb}$ de carbonato de calcio M200 y 15 ppb de carbonato de calcio M40-100. Como caso base de reciclaje de este fluido, se contempló el riesgo de asentamiento del carbonato. Durante el acceso con el ensamblaje de verificación, se encontró aproximadamente $1000 \mathrm{ft}$ con fluido más liviano hasta $9.1 \mathrm{ppg}$. La emulsión se encontró estable y la degradación del fluido fue mínima.

Como ya se mencionó, la primera parte de la estrategia de perforación comprendió el uso de un sistema de cuchara para hueco revestido "Cased hole Whipstock Trackmaster 11 3/4" de uso poco frecuente en el mundo y rediseñada para esta operación en particular. La figura 25 muestra el ensamblaje de corrida del Whipstock. Como puede verse, se utilizó una junta de HWDP por encima de la herramienta de corrida, o running tool, donde se presentaría el mayor bending y por ende, amplitud de los ciclos de fuerzas. Además, el sistema Trackmaster, tiene el moledor anclado mediante un pin de cizalla a la cuchara, que permite la molienda en la misma corrida de asentamiento del whipstock.

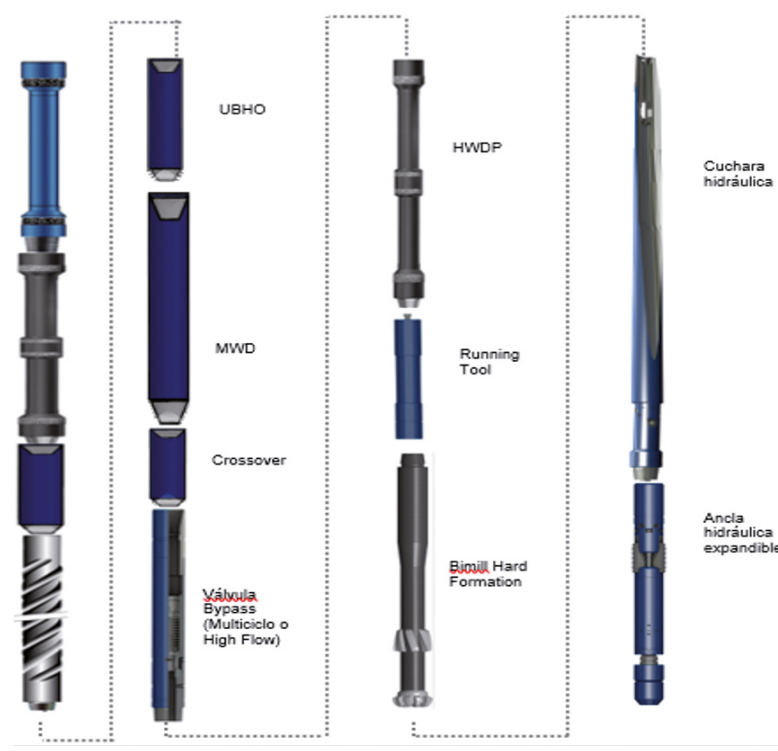

Figura 25. Esquema del ensamblaje usado para asentamiento del whipstock y molienda de la ventana.

Para la molienda de la ventana, se siguió el mapa de parámetros sugerido por la simulación de dinámica de sarta. No obstante, el progreso fue mucho más lento de lo esperado y requirió el aumento progresivo hacia una estrategia más agresiva. Infortunadamente, el moledor PDC se desgastó prematuramente y obligó a realizar 2 corridas adicionales. La figura 26 muestra el desempeño esperado según los trabajos parecidos hechos en el pasado, haciendo la claridad que era el primer trabajo desde un revestimiento de 11 3/4" de grado $\mathrm{P} 110 \mathrm{HC}$.

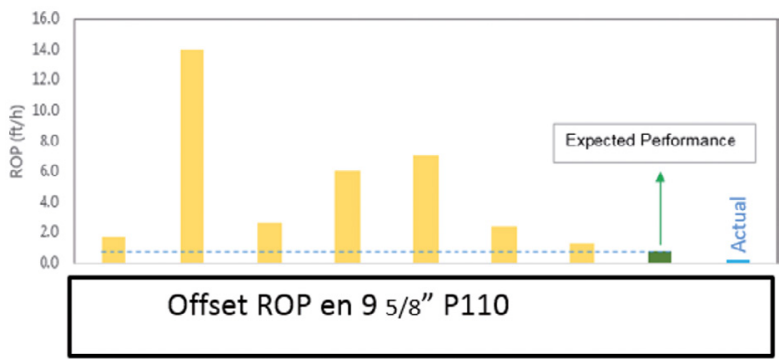

Figura 26. Rendimiento en la molienda de la ventana.

Debido al bajo desempeño del ensamblaje de molienda con moledor PDC, se decidió correr un segundo ensamblaje con un moledor de ventana de carburo de tungsteno que perforó hasta la media rampa. Este último, se desgastó también prematuramente, a razón de la abrasividad de la formación. La figura 27 muestra el desgaste de los mill de las corridas 1 y 2 y los trabajos realizados por cada uno de ellos. La figura 28 muestra el mapa de parámetros usado en la corrida inicial, la cual significó un muy bajo desempeño.

A.

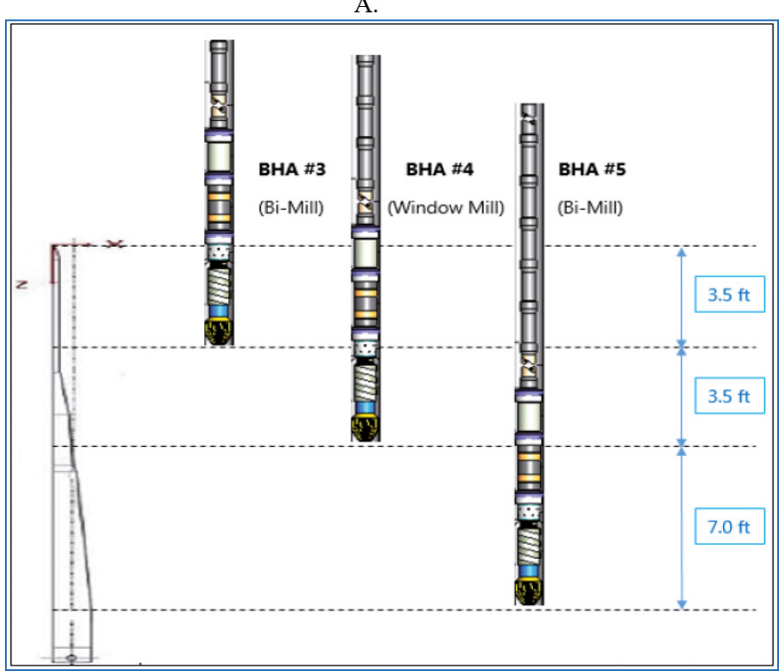

B.

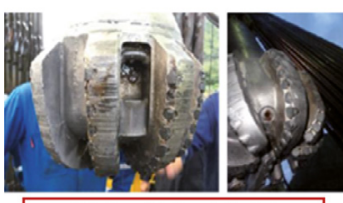

PDC mill Corrida \#1

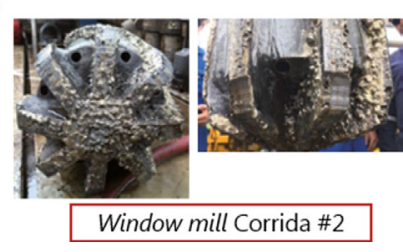

Figura 27. A. Trabajos de las 3 corridas de mill. B. Desgaste de los mill de las corridas 1 y 2 . 


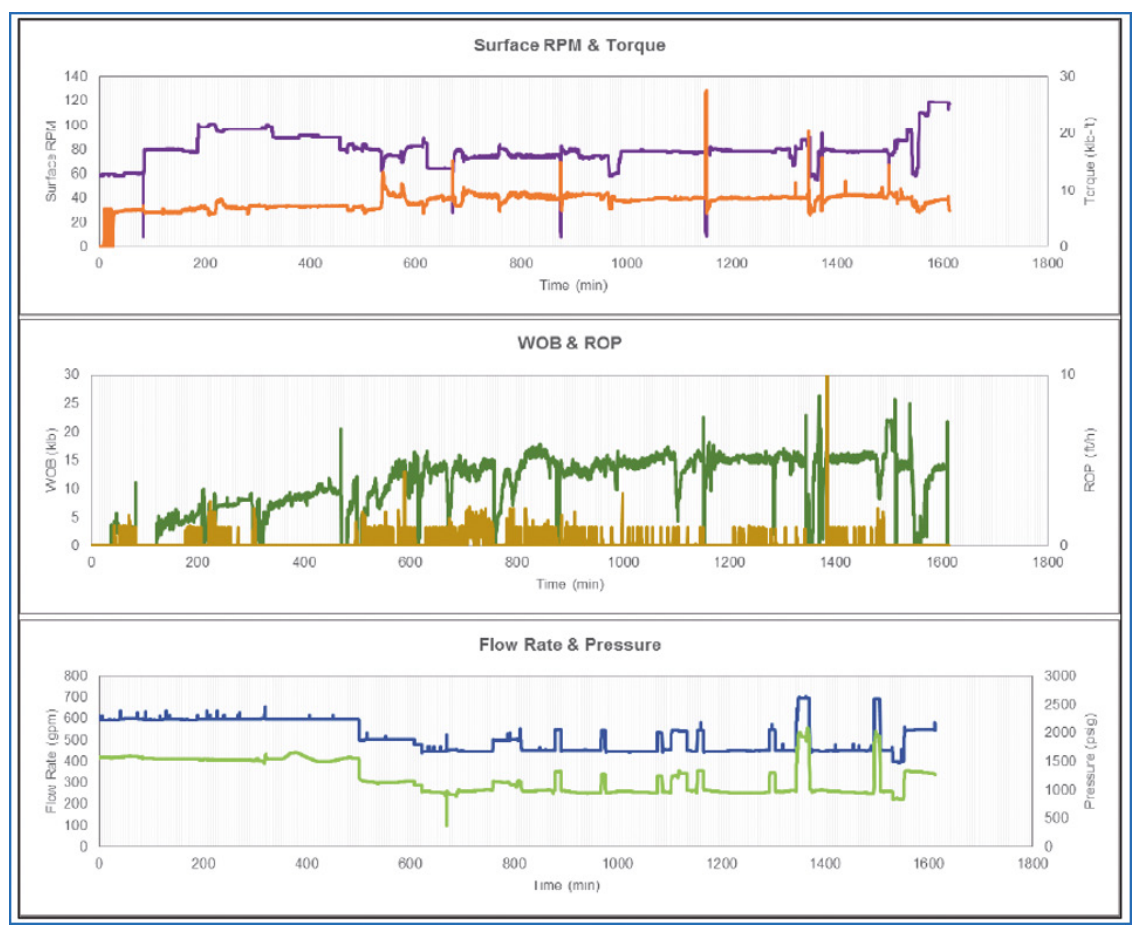

Figura 28. Mapa de parámetros usados para la molienda de la ventana con el BHA\#3. Convención de colores: Púrpura, verde y azul: RPM, WOB y Caudal respectivamente.

Como lecciones aprendidas de la apertura de ventana en casing de 11 3/4" P110HC:

- No existe segregación de las fases del fluido OBM 10.2 ppg tipo yacimiento después de 1.7 años de suspensión. Se observa degradación mínima (1000 ft de evidencia con 9.1 ppg).

- La protección de la sarta usando protectores no rotativos fue efectiva para disminuir el desgaste del revestimiento.

- Es una buena práctica el uso de UBHO en la sarta de corrida del whipstock, como contingencia para orientar la cuchara en caso de daño de la herramienta MWD.

- Es una buena práctica para aumentar el rendimiento, la reducción de la fuerza generada por bomba (pump-off force) mediante la reducción del caudal. La limpieza, puede compensarse mediante el bombeo de trenes de píldora de baja reología seguidos de píldoras viscosas.

- Es una buena práctica la verificación de los protectores no rotativos después del trabajo de molienda, por posible acumulación de cortes de metal en las camisas metálicas de los protectores.
- El uso de la junta de HWDP detrás del moledor dual, permite mitigar el riesgo de falla por rompimiento o twist-off por flexión y/o fatiga.

- Los criterios de aceptación de la calidad de la ventana para este tamaño, mostraron ser efectivos para no tener restricciones en las siguientes corridas de BHA. Para el moledor, 0.25 pulgadas. Para el moledor secundario, 0.5 pulgadas.

- El uso de 8 magnetos de alto poder en el sistema de superficie, permitió recuperar efectivamente el metal cortado de la ventana.

- Se recomienda el re-diseño del moledor PDC que está destinado a la interacción con la formación abrasiva; mediante la inclusión de insertos de carburo de tungsteno (TCI), para hacerlo más agresivo contra el metal y evitar el bajo desempeño. La figura 29 muestra el diseño de moledor híbrido que se hizo para este proyecto, posterior a la primera corrida de molienda.

- Se recomienda reevaluar el riesgo de bajo desempeño, en conjunto con la dinámica de sarta. Para este tamaño, es probable que se necesite operar en condiciones de dinámica no ideales, con el fin de ganar un progreso aceptable. 


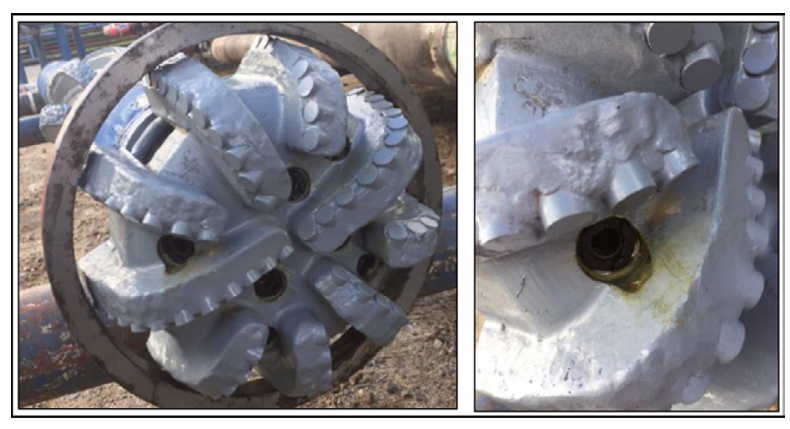

Figura 29. Diseño de moledor híbrido para molienda de la ventana con formación abrasiva detrás.

La perforación de la zona de formaciones suprayacentes al yacimiento (overburden) a través de la falla, girando a la dirección cross-dip se dio sin ningún inconveniente utilizando brocas PDC de cortadores rotativos y RSS. No obstante, debido a la alta abrasión de los intervalos arenosos, las brocas que perforaron estos intervalos sufrieron anillamiento. Es típico tener brocas con anillamiento en estas formaciones. No obstante, su identificación oportuna para la definición del fin de las corridas, es crítico para mantener la integridad de la broca y evitar basura metálica (junk) en el fondo del hueco; que obligaría a trabajos de molienda, pesca, ó incluso, sidetrack operativo.

Otro aspecto a resaltar durante esta perforación, es la presencia de fracturas en las conexiones de las herramientas del BHA sometidas a alto momento de flexión y esfuerzos cíclicos. La figura 30 muestra uno de las fracturas o cracks identificados mediante procedimientos de inspección. Cabe resaltar que no se identificó ningún tipo de fractura en las zonas aledañas a las juntas de tubería.

Se resalta la calidad de la geometría del hueco a partir de los siguientes factores:

- Mitigación de la posible inestabilidad mediante la estrategia de sello usada.

- Disminución de la cantidad de repasos con rotación en cada parada.

- Uso de copilot para el monitoreo del BM/BMTF y disminución de la tortuosidad del hueco.

Algunas lecciones aprendidas de la perforación hasta el punto de revestimiento:

- La estrategia de uso de broca tricone con el diseño seleccionado con motor de fondo demostró ser efectiva para completar la desviación del hueco original en roca abrasiva.
- El monitoreo de las vibraciones tangenciales y el comportamiento de la presión en fondo, demostró ser efectivo para identificar oportunamente el desgaste de las brocas y llamar el fin de las corridas.

- El monitoreo del BM/BMTF demostró ser importante para la construcción de un hueco con baja tortuosidad y para mantener un buen control direccional de la trayectoria.

- Para las herramientas en el BHA: 5 7/8" HWDP S-135 Conexión XT57 y 8 1/4" Drill collars 100K Conexión $65 / 8$ " Reg, se recomienda el procedimiento riguroso de inspección instaurado en este proyecto, sugerido además en el standard DS1 de TH-Hill; el cambio de las herramientas pasando por altos DLS y la trazabilidad de la cantidad de rotación acumulada de las herramientas sometidas a alta flexión.

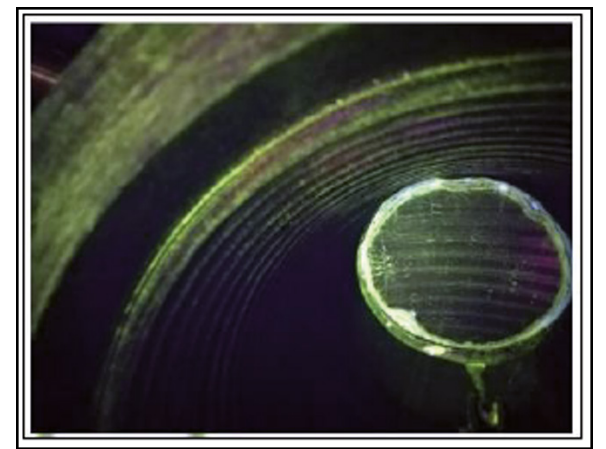

Figura 30. Crack identificado durante la inspección de uno de los ensamblajes de perforación.

Se hizo una corrida dedicada para tocar el yacimiento, con las siguientes observaciones:

- Existe un riesgo relevante de pérdidas en la formación yacimiento, donde se definiría el fin de la sección, debido a la permeabilidad y la posición estructural del pozo (el pozo atraviesa la cresta de la estructura); lo cual incrementaba la probabilidad de presencia de fracturas naturales. Adicional a lo anterior, con el fin de estabilizar la zona por debajo de la falla, se usó un lodo de $12.0 \mathrm{ppg}$. La magnitud del esfuerzo mínimo en la formación yacimiento, se estimaba en 14.0 ppg con bastante incertidumbre.

- El asentamiento del revestimiento, cubriendo la zona por debajo la falla hasta el yacimiento, era crítico para iniciar la sección de 8 1/2” con lodo de yacimiento (Sin asfaltos, grafitos ni nanopartículas) de bajo peso. 
- Se armó un ensamblaje sencillo (Rotario) para tocar el yacimiento, con el fin de tener margen de trabajo y bombeo de material de control de pérdidas.

- Se armó un MWD con probeta espaciada, con el fin de acercar el sensor de rayos gamma lo máximo posible a la broca.

El trabajo de revestimiento se hizo sin problemas relevantes, a pesar del perfil exigente del pozo y del tamaño del espacio anular (Liner de $95 / 8$ " en hueco de $105 / 8$ ”). Se utilizó un equipo de tope de liner robusto que permitía el trabajo de la sarta con zapato rimador, con el fin de superar la complejidad geométrica del hueco, en caso de presentarse. La capacidad de torque, tanto de la herramienta de corrida, como de la tubería de $95 / 8$ " y la tubería de perforación, permitían trabajar cómodamente con rotación, en caso de requerirse. No obstante, debido a la alta calidad de hueco, no fue necesario rotar, ni siquiera por debajo de la falla, para llevar este liner a fondo.

Para la perforación de la sección de yacimiento ( 8 1/2”), se resaltan los siguientes aspectos:

- Se utilizó una broca PDC versátil (8 aletas / cortadores $16 \mathrm{~mm}$ ) para la perforación del equipo de flotación y la perforación del yacimiento (Con alta resistencia compresiva y abrasión), con muy buen desempeño.

- El uso de registro de rayos gamma azimutal para la reinterpretación permanente del modelo estructural.

- La reevaluación permanente del modelo de estabilidad, de acuerdo a la litología encontrada, con el fin de la modificación en avanzada de la estrategia de sello y de peso del fluido de perforación.

- Debido a la incertidumbre en la presión de poro en los yacimientos a buscar, por tratarse de un pozo exploratorio, se realizó simulacros de control de pozo dedicados, bajo la supervisión de expertos, incluyendo $12 \mathrm{hrs}$ de stripping (paso de tubería hacia el hueco con el preventor anular cerrado).

Análisis de desempeño. Gracias al diseño robusto $\mathrm{y}$ al aseguramiento de integridad realizado para este proyecto, se logró un desempeño excepcional de sólo $7 \%$ de NPT (Tiempo no productivo). Se resalta, que de este porcentaje, $0 \%$ corresponde a problemas del hueco, debido a la alta calidad de construcción del hueco. La figura 31 muestra un resumen de la distribución del $7 \%$ de NPT.
Algo adicional que vale la pena mencionar, es la capitalización del activo intangible y su aprovechamiento para este proyecto, como parte de la madurez organizacional de la compañía operadora. Durante la ejecución de este proyecto de estudio, además, se generaron cincuenta y dos (52) proyectos de mejoramiento continuo, distribuidos como lo muestra la figura 32 .

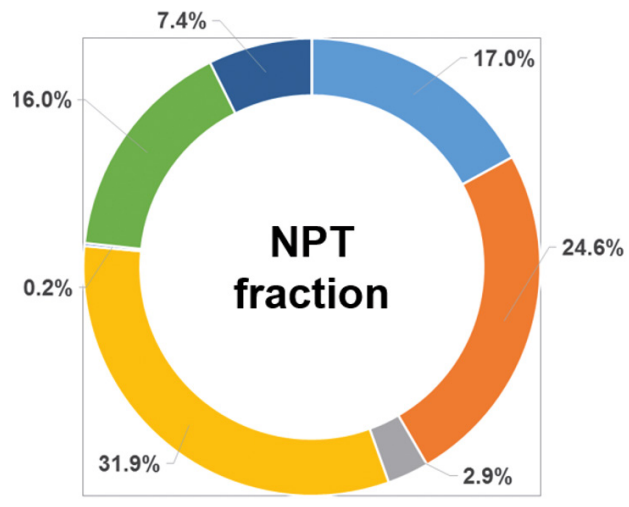

$$
\begin{array}{ll}
\text { " Rig Repair } & \text { " Fitting Failure } \\
\text { = Milling Performance } & \text { " Directional Tools Failure } \\
\text { - Surface Equipment Failure (Not rig) } & \text { " Weather } \\
\text { = Community / Entorno } & \text { " Hole Condition }
\end{array}
$$

Figura 31. Distribución del NPT.

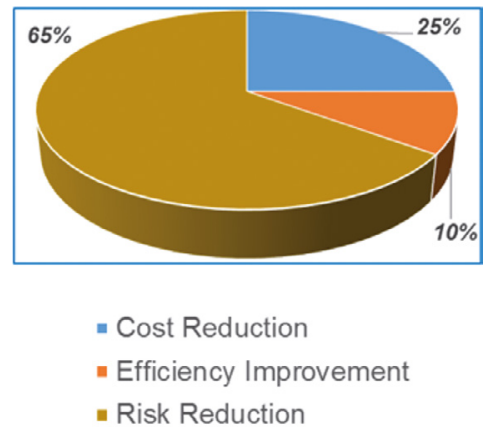

Figura 32. Distribución de los proyectos de mejoramiento contínuo.

En comparación con el plan, el buen rendimiento del proyecto generó un valor ganado de 1.7 millones de dólares.

\section{Conclusiones}

- Los proyectos de reacceso desde los revestimientos intermedios en pozos existentes son una alternativa a pozos de superficie, para proyectos de exploración en zonas de baja resolución sísmica e incertidumbre de los WOC.

- Los proyectos de reacceso en la cuenca sub-andina requieren una rigurosidad de ingeniería, disciplina 
operacional y aseguramiento de integridad, aterrizados al contexto específico del proyecto. Algunos de los aspectos relevantes son:

- Selección del punto de asentamiento de la cuchara de desviación.

- Diseño de la cuchara y del moledor usado para abrir la ventana, además del procedimiento

- Método de completamiento del sidetrack del pozo original, teniendo en cuenta el momento de flexión y ciclos de esfuerzos a los que están sometidos las herramientas pasando por alto DLS.

- Selección de broca y herramientas direccionales para la construcción de la trayectoria.

- Evaluación del modelo de estabilidad y severidad de trayectoria para el diseño del fluido de perforación.

- Monitoreo del BM/BMTF y diseño de procedimientos para garantizar la calidad de la geometría del hueco.

- Procedimientos y diseños específicos para contexto de incertidumbre geológica y en términos del modelo de estabilidad (Magnitud del esfuerzo mínimo y presión de poro).

- Aseguramiento en términos de seguridad de procesos a personas, procedimientos, planta $\mathrm{y}$ equipos.

- Es muy importante la capitalización del activo intangible (Lecciones aprendidas) para la construcción progresiva de la madurez organizacional.

\section{Reconocimientos}

Los autores agradecen la disposición de Equión-Energía y el grupo de exploración de Niscota, de permitir el uso de la información en la cual se fundamenta este artículo. Consideramos que es un aporte importante para el crecimiento de la industria en Colombia y el mundo.

\section{Referencias}

ANH. (2007). Colombian Sedimentary Basins: Nomenclature, boundaries and Petroleum Geology, a New Proposal. In Agencia Nacional de Hidrocarburos - A.N.H.- (Issues 978-95898237-0-5). http://www.anh.gov.co/InformacionGeologica-y-Geofisica/Cuencas-sedimentarias/ Documents/colombian_sedimentary_basins.pdf
API. (1981). API Recommended practice for drill stem design and operating limits. American Petroleum Institute.

Contreras, O., Hareland, G., Husein, M., Nygaard, R., \& Alsaba, M. (2014). Experimental Investigation on Wellbore Strengthening in Shales by Means of Nanoparticle-Based Drilling Fluids . https://doi. org/10.2118/170589-MS

Hoelscher, K. P., Young, S., Friedheim, J., \& De Stefano, G. (2013). Nanotechnology Application in Drilling Fluids .

Lubinski, A. (1961). Maximum Permissible DogLegs in Rotary Boreholes. Journal of Petroleum Technology, 13(02), 175-194. https://doi. org/10.2118/1543-G-PA

Shokir, E. (2004). A novel pc program for drill string failure detection and prevention before and while drilling specially in new areas. Oil \& Gas Business, 1-14.

Stelzer, C. (2007). Drillpipe Failure and its Prediction. Mining University Leoben.

Teng, X., Yang, P., Li, N., Yu, F., Jin, Y., \& Chen, M. (2015). Successful HPHT Drilling Through Innovative Practices: Sharing the Subsalt HPHT Well Drilling Case in Tarim Basin. https://doi.org/ SPE-172782-MS

Ugochukwu, O., Okubor, E., Aigbedion, P., \& Ayodele, A. (2016). Optimizing Whipstock Sidetrack Operations. https://doi.org/10.2118/184259-MS

Vaisberg, O., Vincke, O., Perrin, G., Sarda, J. P., \& Fay, J. B. (2002). Fatigue of Drillstring: State of the Art. Oil \& Gas Science and Technology, 57(1), 7-37. https://doi.org/10.2516/ogst:2002002

Zhang, Y., Burhan, Y., Chen, C., Tammineni, S., Durairajan, B., Mathanagopalan, S., \& Ford, R. (2013). Fully Rotating PDC Cutter Gaining Momentum: Conquering Frictional Heat in Hard/ Abrasive Formations Improves Drilling Efficiency. https://doi.org/10.2118/166465-MS

Fecha de recepción: 28 de abril de 2020

Fecha de aceptación: 11 de junio de 2021 